\title{
Análisis de la trazabilidad en los valores del AOD obtenidos a partir de las medidas de las redes AERONET-CIMEL y GAW-PFR durante el período 2005-2015 en el Observatorio Atmosférico de Izaña
}

Nota técnica 23 de AEMET

\section{Pedro Miguel Romero Campos ${ }^{(1)}$ Emilio Cuevas Agulló (1) Stelios Kazadzis ${ }^{(2)}$ Natalia Kouremeti ${ }^{(2)}$ Rosa Delia García Cabrera ${ }^{(3)}$ Carmen Guirado Fuentes ${ }^{(4)}$}

(1) Centro de Investigación Atmosférica de Izaña. Agencia Estatal de Meteorología. Santa Cruz de Tenerife. 38001. España.

(2) Physikalisch-Meteorologisches Observatorium Davos, World Radiation Center. Davos. CH-7260. Suiza.

(3) Air Liquide España. Delegación Canarias. Santa Cruz de Tenerife. Candelaria. 38509. España.

(4) Grupo de Óptica Atmosférica. Universidad de Valladolid. Valladolid. 47011. España.

Centro de Investigación Atmosférica de Izaña Agencia Estatal de Meteorología (AEMET) 


\section{datos \\ abiertos}

Aviso Legal: los contenidos de esta publicación podrán ser reutilizados, citando la fuente y la fecha, en su caso, de la última actualización

\section{Edita:}

(C) Ministerio de Agricultura y Pesca, Alimentación y Medio Ambiente Agencia Estatal de Meteorología Madrid, 2017

Catálogo de Publicaciones de la Administración General del Estado: https://cpage.mpr.gob.es

NIPO: 014-17-008-X

https://doi.org/10.31978/014-17-008-X

Agencia Estatal de Meteorología (AEMET)

C/ Leonardo Prieto Castro, 8

28040 Madrid

http://www.aemet.es/

@Aemet_Esp 


\section{Índice}

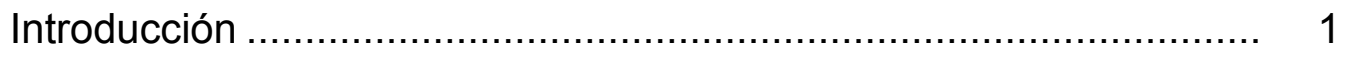

1. Estudio de la trazabilidad del AOD ..................................... 2

2. Análisis de la no trazabilidad ............................................ 6

2.1. Influencia de la calibración y del cálculo del AOD............ 6

2.2. Influencia de variables de entrada en el AOD ................ 9

2.2.1. Influencia de la dispersión Rayleigh .................... 10

2.2.2. Influencia de la absorción del ozono ................... 12

2.2.3. Influencia de la absorción del NO2 ..................... 13

2.2.4. Efecto de la combinación de componentes ........... 15

2.3. Influencia de la nubosidad ................................... 17

2.4. Influencia de otros factores ................................. 20

3. Conclusiones y recomendaciones ................................. 20

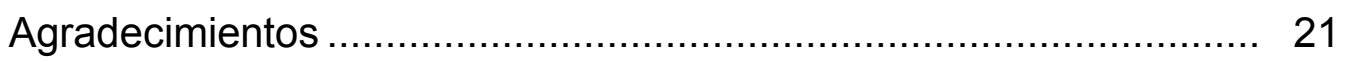

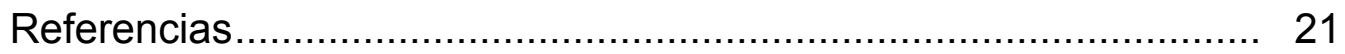





\section{Introducción}

Hoy en día, existen dos grandes redes mundiales de fotómetros terrestres para la medida de las propiedades ópticas del aerosol en la columna atmosférica. Se trata de las redes AERONET-CIMEL (AErosol RObotic NETwork - CIMEL Electronique sun-photometer; http://aeronet.gsfc.nasa.gov) y GAW-PFR (Global Atmospheric Watch - Precision Filter Radiometer; http://www.pmodwrc.ch/worcc/).

La red GAW-PFR (Wehrli, 2005), fue diseñada para el análisis mundial a largo plazo del aerosol atmosférico de fondo y detección de tendencias de aerosoles a largo plazo en atmósferas limpias. Por este motivo, esta red utiliza instrumentos muy estables y de alta precisión (PFR - Precision Filter Radiometer, (Wehrli, 2008)) que han sido instalados en lugares remotos alrededor del mundo representativos de las condiciones de background atmosférico. Sin embargo, la red AERONET-CIMEL (Holben et al., 1998), ha sido primariamente diseñada para validar productos satelitales y caracterizar el aerosol global a partir de sus propiedades ópticas en diferentes lugares y altitudes alrededor del mundo donde se producen condiciones medioambientales y de tiempo muy distintas que abarcan un amplio rango de tipos, tamaños y concentraciones de aerosoles atmosféricos. Ambas redes, aunque diseñadas para cubrir objetivos distintos, sirven como referencias mundiales en el estudio de las propiedades ópticas de aerosoles y como herramientas en la validación de observaciones a bordo de satélites y modelos.

Son muy pocos los lugares en el mundo en los que se dispone de observaciones simultáneas de estas dos redes, ya que, esto implica que en dichas estaciones, deben darse las condiciones atmosféricas representativas para caracterizar al aerosol en todas aquellas situaciones meteorológicas diferentes para las que las redes fueron diseñadas.

En este sentido, el Observatorio Atmosférico de Izaña (IZO, $28.31^{\circ} \mathrm{N}, 16.50^{\circ} \mathrm{W}, 2370$ m.s.n.m.), gestionado por el Centro de Investigación Atmosférica de Izaña (CIAl; http://izana.aemet.es, perteneciente a la Agencia Estatal de Meteorología (AEMET; http://www.aemet.es), es un lugar ideal para la comparación y el estudio a largo plazo de propiedades ópticas de aerosol obtenidas con instrumentación, y bajo condiciones y escenarios meteorológicos, muy diferentes. Esto es debido a que IZO está localizado en la troposfera libre (FT) sobre Tenerife, lo cual favorece, durante la mayor parte del año, la presencia de cielos muy limpios representativos de condiciones de fondo atmosférico. Por otro lado, su proximidad al continente africano lo convierte en un lugar de observación y caracterización de la capa de aire sahariana que presenta alto contenido de polvo mineral del desierto, especialmente durante los meses de verano, los cuales, contienen altos niveles de carga de aerosol (Basart et al., 2009; Rodríguez et al., 20I I; Cuevas et al., 20I3). Esta situación privilegiada de IZO lo han convertido, desde 200I y 2003, respectivamente, en un lugar de calibración de las redes GAW-PFR y AERONET-CIMEL donde las constantes extraterrestres aparentes son determinadas mediante observaciones directas al sol (técnica Langley). Además, desde julio de 20I4, IZO ha sido también designado por la Comisión para Instrumentos y Métodos de Observación (CIMO) de la Organización Meteorológica Mundial (OMM) como banco de pruebas (Testbed) para instrumentos de teledetección de vapor de agua y aerosoles.

Dado que las redes AERONET-CIMEL y GAW-PFR son diferentes en cuanto a tipos, características, número y localización de fotómetros utilizados, así como a metodologías, procedimientos de calibración y algoritmos de cálculo empleados en la evaluación de datos, sería conveniente comparar los productos similares suministrados por ambas redes y así determinar en qué medida son homogéneos o equivalentes.

Como contribución a esta tarea, en este trabajo nos proponemos el objetivo de analizar las diferencias que existen en una propiedad óptica, fundamental para conocer y caracterizar el contenido en columna de los aerosoles atmosféricos, que es el Espesor Óptico de Aerosoles (AOD) obtenido para diferentes longitudes de onda por cada una de las dos redes. Esta propiedad mide la extinción de la irradiancia directa normal procedente del disco solar debida, exclusivamente, a la presencia de los aerosoles. Para ello, se han comparado entre sí los productos de máxima calidad de AOD obtenidos en IZO entre 2005 y 2015 por los fotómetros de una y otra red.

El análisis se estructura en tres capítulos. En el capítulo I, se expone el concepto de trazabilidad y se explica en qué se basa la definición y cuáles son sus implicaciones. Posteriormente, se aplica a las series de AOD y se muestran los resultados. En el capítulo 2, se analizan las principales causas que pueden dar lugar a diferencias no trazables de AOD para cada una de las parejas de series comparadas en cada longitud de onda. Finalmente, el capítulo 3, recoge las conclusiones y recomendaciones para mejorar, en la medida de lo posible, la trazabilidad y la homogeneidad de estos productos. 


\section{Capítulo I. Estudio de la trazabilidad del AOD}

Basándose en el Comité Conjunto para Guías en Metrología (JCGM), la Comisión para Instrumentos y Métodos de Observación (CIMO) de la Organización Meteorológica Mundial (OMM) define la trazabilidad entre medidas (WMO, 2014) como "aquella propiedad que nos permite relacionar con una referencia dada, ya sea un estándar nacional o internacional, el resultado obtenido de una medida a través de una cadena ininterrumpida de comparaciones con incertidumbres establecidas". Por tanto, lo que nos permite conocer la trazabilidad es el seguimiento o rastreo de los valores de las medidas conociendo su historial, comparaciones, calibraciones e incertidumbres asociadas y poder así hacerlas comparables respecto de una referencia dada.

En el caso que nos ocupa, nuestra variable meteorológica es el AOD. Los límites de incertidumbre propuestos por la OMM para el estudio de la trazabilidad del AOD tienen en cuenta, por un lado, la incertidumbre propia de los cálculos de esta variable, y por otro, la incertidumbre asociada a la calibración de los equipos que se basan en medidas de transmisión finita (como los fotómetros PFR y CIMEL), la cual, viene dada por la señal supuestamente medida en la parte superior de la atmósfera (señal extraterrestre) dividida por la masa óptica (WMO/GAW, 2005). Dado que la máxima incertidumbre esperada en los cálculos del AOD es de \pm 0.01 y para la calibración de los equipos es del $1 \%$, los límites de incertidumbre al 95\% para la trazabilidad del AOD establecidos por la OMM en este tipo de instrumentos se definen de la siguiente manera (WMO/GAW, 2005):

$$
U_{95}= \pm(0.005+0.010 / m)
$$

donde $m$ es la masa óptica relativa.

Para la red GAW-PFR, la incertidumbre obtenida en el AOD a partir de las medidas en cualquiera de sus canales es del orden de \pm 0.0 I (Wehrli, 2000), y la del AOD de nivel 2 de AERONET-CIMEL (valores de alta calidad, filtrados por nubes y visualmente inspeccionados), se encuentra entre 0.01 y 0.02 bajo condiciones de cielos despejados (Eck et al., 1999; Barreto et al., 2016). Una y otra red son, pues, candidatas a cumplir los requerimientos de calidad establecidos por la OMM para el AOD.

La definición de trazabilidad dada por la expresión (I) nos da el máximo error permitido en la determinación del AOD para garantizar la calidad de dicha variable así como la equivalencia entre medidas similares procedentes de distintas fuentes, y ha de cumplirse, al menos, para el $95 \%$ de los valores de la población en estudio. Cuando esta expresión se aplica a dos valores de AOD simultáneos obtenidos a partir de las medidas en canales similares de equipos diferentes, nos permitirá conocer si forman parte o no de la misma muestra homogénea, y por tanto, si son o no equivalentes, dependiendo de si su diferencia se encuentra o no dentro de los límites de incertidumbre marcados por dicha expresión. Este es el estudio que abordamos en este trabajo en el que comparamos los valores de AOD para cuatro canales de las redes AERONET-CIMEL y GAW-PFR en el período 2005 a 2015 durante las medidas simultáneas realizadas en el Observatorio Atmosférico de Izaña. Analizaremos en qué grado se cumple la trazabilidad entre ambas redes así como las causas probables que puedan influir en la no trazabilidad.

El primer factor de la expresión (I), es decir, \pm 0.005 , representa el error máximo deseable debido al propio cálculo del AOD. En este error, irían implícitos los errores instrumentales y los de todos los parámetros o variables que intervienen en la fórmula de cálculo del AOD, así como la propagación de los mismos. Si no hubiera más errores añadidos estaríamos diciendo con ello que el valor verdadero de AOD debería encontrarse en un rango de amplitud máxima de 0.01 en torno al valor de AOD calculado, es decir:

$$
A O D_{\text {verdadero }}=A O D_{\text {calculado }} \pm 0.005 \text {. }
$$


Sin embargo, también nos encontramos en la práctica con el error de calibración de los equipos (fotómetros en nuestro caso). Este error añadido es el que viene dado por el segundo factor de la expresión (I). La OMM ha establecido que el error de una calibración de calidad debería ser como máximo del $1 \%$. A partir de un sencillo cálculo matemático podemos ver que ese $1 \%$ se traduce en el factor $\pm 0.010 / \mathrm{m}$.

En efecto, ley de Bouguer-Lambert-Beer, en su expresión logarítmica y considerando una misma masa óptica para todos los componentes atmosféricos que intervienen en la extinción, establece que:

$$
A O D^{\prime}=\left[\frac{\ln \left(I_{0}^{\prime}\right)-\ln (I)}{m}-\sum_{i=1}^{n} \delta_{i}\right] \pm 0.005
$$

Donde $I_{0}^{\prime}$ es la señal extraterrestre (constante de calibración), $I$ es la intensidad medida por el fotómetro, $m$ es la masa óptica relativa y $\sum_{i=1}^{n} \delta_{i}$ representa la suma o contribuciones a la extinción total del resto de componentes atmosféricos distintos a los aerosoles (moléculas de aire, ozono, NO2, etc.). El factor \pm 0.005 es el máximo error permitido en el propio cálculo de $A O D^{\prime}$. Un error de calibración del $1 \%$ en $I_{0}^{\prime}$ significaría que esta, podría ponerse en función de la verdadera constante de calibración $I_{0}$, como:

$$
I_{0}^{\prime}=I_{0} \pm \frac{I_{0}}{100}=I_{0} \pm 0.01 \times I_{0}=(1 \pm 0.01) \times I_{0}=k \times I_{0}
$$

donde $I_{0}$ sería el valor de la constante de calibración sin el error del $\mathrm{l} \%$. Substituyendo $I_{0}^{\prime}=k \times I_{0}$ en (2) resulta que:

$$
\begin{aligned}
& A O D^{\prime}=\left[\frac{\ln \left(I_{0}^{\prime}\right)-\ln (I)}{m}-\sum_{i=1}^{n} \delta_{i}\right] \pm 0.005=\left[\frac{\ln \left(k \times I_{0}\right)-\ln (I)}{m}-\sum_{i=1}^{n} \delta_{i}\right] \pm 0.005= \\
& =\left[\frac{\ln k+\ln \left(I_{0}\right)-\ln (I)}{m}-\sum_{i=1}^{n} \delta_{i}\right] \pm 0.005=A O D+\frac{\ln k}{m} \pm 0.005
\end{aligned}
$$

Donde $A O D=\frac{\ln \left(I_{0}\right)-\ln (I)}{m}-\sum_{i=1}^{n} \delta_{i}$ es el AOD sin el error de calibración y sin el error propagado en el propio cálculo a partir de los errores instrumentales, etc. Substituyendo $k=1 \pm 0.01$ en (3), y considerando el doble signo \pm , la expresión (3) quedaría como:

$$
A O D^{\prime}=A O D+\frac{\ln (1 \pm 0.01)}{m} \pm 0.005=A O D \pm\left(0.005+\frac{0.010}{m}\right)=A O D+U_{95} .
$$

De aquí que:

$$
A O D^{\prime}-A O D=U_{95}
$$

Las expresiones (I) y (4) son generales y deben cumplirse para cualesquiera valores de AOD, independientemente de la longitud de onda de trabajo y de la masa óptica asociada al instante en el que se toman las medidas. Por tanto, para que dos medidas de AOD realizadas en canales similares y por equipos diferentes sean trazables y de la calidad exigida por la OMM, la diferencia entre ellas debe ser como máximo el valor de trazabilidad definido por $U_{95}$ para la masa óptica asociada a dichas medidas. 
Cada valor particular de masa óptica define una incertidumbre máxima diferente para que se cumpla la trazabilidad. La mayor incertidumbre máxima permitida por (I) se daría en el caso de que midiéramos el $A O D$ en el mismo cénit (ángulo cenital 0 ) y valdría \pm 0.015 ya que el valor para la masa óptica relativa sería de I. En este caso, el valor verdadero del AOD, o la diferencia entre dos valores de AOD en el caso de una comparación, debería encontrarse en un rango de amplitud máxima de 0.03 centrado sobre el valor calculado o sobre la diferencia de los mismos, respectivamente.

Los fotómetros GAW-PFR y AERONET-CIMEL, están diseñados para realizar medidas de transmisión finita de la irradiancia solar directa normal con campos de visión angulares (FOV) pequeños y canales de banda estrecha distribuidos en los rangos visible e infrarrojo cercano del espectro solar. Algunas de las características más relevantes de estos equipos relacionadas con el estudio que se aborda se resumen en la Tabla $\mathrm{I}$.

\begin{tabular}{|c|c|c|c|c|}
\hline IZO (2005- 2015) & Número de Instrumento & $\begin{array}{c}\text { Longitudes de onda analizadas } \\
(\mathbf{n m})\end{array}$ & $\begin{array}{c}\text { FOV } \\
(\mathbf{(})\end{array}$ & $\begin{array}{c}\text { FWHM } \\
(\mathbf{n m})\end{array}$ \\
\hline GAW-PFR & $6,21,25$ & $368,412,500,862$ & 2.5 & 5.0 \\
\hline AERONET-CIMEL & $\begin{array}{l}25,44,45,79,117, \\
140,244,245,347,380, \\
382,383,398,421,548\end{array}$ & $380,440,500,870$ & 1.2 & 10.0 \\
\hline
\end{tabular}

Tabla I: Principales características de los instrumentos comparados en IZO de 2005 a 20 I5. FOV es el campo de visión y FWHM es el ancho de banda a media altura de los canales analizados.

La comparación se ha llevado a cabo sólo entre los canales más próximos de ambas redes de fotómetros de los que se disponen de medidas. Así, para el caso de GAW-PFR se analizaron los 4 canales disponibles de $862 \mathrm{~nm}$, $500 \mathrm{~nm}, 4 \mathrm{I} 2 \mathrm{~nm}$ y $368 \mathrm{~nm}$, y en el caso de AERONET-CIMEL se analizaron los canales de $870 \mathrm{~nm}, 500 \mathrm{~nm}$, 440nm y $380 \mathrm{~nm}$. Para 500nm, las longitudes de onda nominales de los canales comparados de ambas redes en el período [2005 - 2015] difieren como máximo en I.8nm. Sin embargo, las diferencias de longitudes de onda nominales entre el resto de canales similares comparados son mayores. Estos valores oscilan en los intervalos [6nm, 9nm], [26nm, $28.8 \mathrm{~nm}]$ y $[\mathrm{IInm}, 13.5 \mathrm{~nm}]$ para los canales de [870nm, 862nm], [440nm, 4I2nm] y [380nm, 368nm], respectivamente. Este hecho, podría influir, de forma no despreciable, en los valores de AOD comparados. Por ello, hemos extrapolado el AOD de los canales originales de GAW-PFR a las longitudes de onda exactas de los canales AERONET-CIMEL con los que se comparan en cada instante haciendo uso de los parámetros de Angstrom obtenidos por GAW-PFR. Estos parámetros, de los que hablaremos más tarde, son el coeficiente $\beta$ y el exponente $\alpha$ de la expresión:

$$
A O D(\lambda)=\beta \times \lambda^{-\alpha}
$$

Que constituye la ley de Angstrom, la cual, nos permite calcular el AOD a diferentes longitudes de onda y relacionar entre sí los calculados para el mismo instante de tiempo.

El cruce de datos en nuestro análisis de comparación se ha realizado con valores de AOD de ambas redes obtenidos en el mismo minuto y ofrecidos oficialmente como datos de máxima calidad por cada una de las redes. Por eso, en el caso de GAW-PFR (Wehrli, 2008), se han seleccionado únicamente aquellos valores de espesor óptico etiquetados con códigos ("flags") que toman el valor 0 , los cuales garantizan que no están afectados por la presencia de nubes. Además, estos datos han sido también visualmente inspeccionados. En el caso de la red AERONET-CIMEL, los datos de AOD seleccionados son los de nivel 2, filtrados de nubes por el algoritmo de Smirnov (Smirnov, 2000) basado en el método de tripletes y depurados también visualmente. Estos datos de AOD AERONET de nivel 2 corresponden a la versión 2 de los algoritmos la cual ha sido, hasta hace poco, la versión más actualizada de trabajo. Actualmente, existe una nueva versión, que es la versión 3 , que se encuentra en fase de pruebas, ejecutándose de forma paralela a la versión 2 y de la que, por el momento, no se dispone del nivel 2 de calidad de datos de AOD. 
Hay que indicar que, aunque trabajemos con datos oficiales de calidad máxima asegurada, los límites de incertidumbre en el cálculo del AOD para la trazabilidad dados por (I) hacen posible que, en días muy limpios donde el AOD es muy bajo, éste pudiera tomar valores nulos o incluso negativos. Como estos valores no tienen sentido físico y no pueden ser usados, matemáticamente, en la obtención de los parámetros de Angstrom, los hemos descartado de nuestro estudio, y en cualquier caso, representan un porcentaje muy pequeño de los datos analizados. Por otro lado, ha habido también registros en los que la medida del AOD, para algún canal concreto, no ha estado disponible por otros motivos. Todo esto hace que no haya el mismo número de diferencias de AOD a analizar para cada pareja de canales comparados en el conjunto de registros comunes cruzados.

En la Figura I, mostramos las diferencias de AOD frente al tiempo entre AERONET-CIMEL y GAW-PFR en IZO para el período 2005 a 2015 de cada una de las parejas de canales comparados.
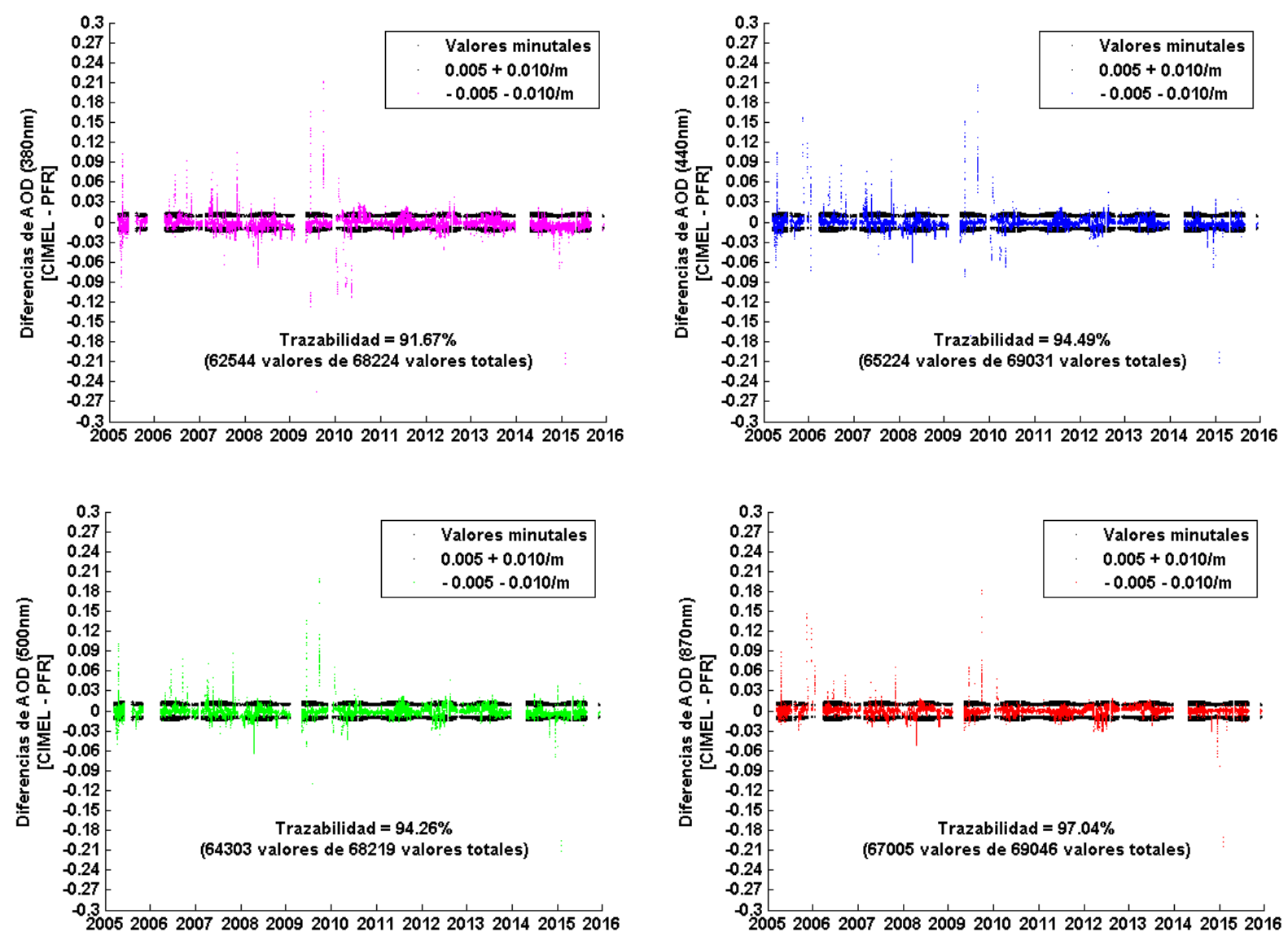

Figura I: Diferencias de AOD entre CIMEL y PFR, según canal. En negro se representan los límites de trazabilidad calculados en función de la masa óptica según la expresión (I). Los canales comparados del PFR se han extrapolado a las correspondientes longitudes de onda CIMEL usando los parámetros de Angstrom obtenidos por GAW-PFR. Observatorio de Izaña, $2005-2015$.

Considerando el período completo, el único canal donde se cumplen los criterios de trazabilidad al $95 \%$ es el canal de $870 \mathrm{~nm}$, donde la trazabilidad es del $97.04 \%$. El canal más afectado por diferencias no trazables es el canal de $380 \mathrm{~nm}$ con un $91.67 \%$ de trazabilidad. Los canales de $440 \mathrm{~nm}$ y $500 \mathrm{~nm}$ toman valores muy próximos al $95 \%$ de trazabilidad, presentando una trazabilidad similar, algo superior al $94 \%$ (94.49\% y $94.26 \%$, respectivamente). Esto significa que, a pesar del uso de metodologías distintas a la hora de calibrar, de filtrar por nubes y de evaluar los AOD por ambas redes de fotómetros, las trazabilidades son muy elevadas incluso añadiendo los errores de propagación debidos a la extrapolación de los AOD de los canales PFR originales a las longitudes de onda CIMEL mediante la aplicación de la ley de Angstrom. 


\section{Capítulo 2. Análisis de la no trazabilidad}

Para analizar los factores que puedan estar afectando a la trazabilidad del AOD, nos apoyamos en la propia definición de límites de incertidumbre dada por la OMM mediante la expresión (I).

En dicha expresión, nos encontramos en dos términos separados, los errores asociados con la incertidumbre en la medida del AOD. Por un lado, tenemos el error derivado en el propio proceso de cálculo, y por otro, el error procedente de la calibración de los equipos. Este último, da cuenta de la diferencia entre el valor usado para obtener el AOD diario (seleccionado de una serie general de valores de calibración para días muy limpios y estables con diferencias menores del $1 \%$ entre ellos) y el que debería haberse utilizado procedente del langley-plot diario, si para ese día, las condiciones meteorológicas nos hubiesen permitido una calibración sin errores significativos (inferiores al $1 \%$ ).

Por tanto, las dos causas principales de la no trazabilidad serían:

I. Una calibración deficiente en alguno de los equipos que se comparan, o en los dos.

2. Diferentes errores en el proceso de cálculo del AOD debidos a:

a. Uso de distintas variables de entrada necesarias para tener en cuenta la extinción debida a otros componentes que no son aerosoles y que habrá que restar de la extinción total para evaluar el AOD. Entre estas variables de entrada se encuentran: la distancia TierraSol del día en cuestión, la elevación solar y la masa óptica de los componentes involucrados, así como los datos de presión y valores de ozono y NO2 en columna procedentes de bases de datos diferentes utilizadas por las dos redes.

b. Señales de mala calidad, o no apropiadas para el cálculo del AOD, debido a excesivo ruido y/ó presencia de obstáculos como pueden ser nubes no detectadas por los algoritmos de filtrado u otros obstáculos de diferente naturaleza como consecuencia de limpiezas de ópticas de entrada y supervisión de apuntamiento de los fotómetros.

\section{I. Influencia de la calibración y del cálculo del AOD}

Los protocolos para los procedimientos de calibración de los fotómetros en AERONET-CIMEL y en GAWPFR son diferentes. Mientras que en el caso de GAW-PFR se establecen calibraciones frecuentes durante todo el año y se interpola linealmente el valor de calibración correspondiente a días en los que no pudo aplicarse el método Langley-plot para su determinación directa, en la red AERONET-CIMEL se asume un valor de calibración constante en el período intermedio entre dos calibraciones consecutivas realizadas con periodicidad anual.

Por ello, no siempre los valores de calibración asignados a días concretos, fuera del período propio de la calibración, son igualmente adecuados a la hora de evaluar el AOD. Una determinación no suficientemente precisa de la constante de calibración influye en la evolución diurna del AOD, y se nota especialmente, en las partes centrales del día, sobretodo, si hay muy poca carga de aerosol y el AOD es muy bajo ( $<0.02$ en torno a $500 \mathrm{~nm}$ ). Pequeñas inexactitudes en la determinación de las constantes extraterrestres no influyen en condiciones atmosféricas normales, pero se hacen evidentes bajos cielos extremadamente limpios en los que el AOD presenta una evolución siguiendo una curva característica de tipo cóncavo o convexo (Romero y Cuevas, 2002; Cachorro et al., 2004).

A continuación, proponemos un método basado en la propia definición de trazabilidad y en la técnica del kciclo (Cachorro et al., 2004) para la determinación de los errores de calibración del AOD que pueden tener lugar en un día cualquiera, con la particularidad, de que aquí, lo usaremos no con el AOD, sino con las diferencias de AOD trazables, lo que le hace más consistente y robusto ya que así se elimina la influencia de la evolución diurna del AOD en el método. Este procedimiento nos permitirá también obtener una estimación del error en el proceso de cálculo del mismo de forma separada al error de calibración. Si en la expresión (4) substituimos la expresión (I), obtenemos que: 


$$
A O D^{\prime}-A O D=U_{95}= \pm\left(0.005+\frac{0.010}{m}\right)
$$

Analizando la expresión (5), observamos que si representamos las diferencias de AOD frente al inverso de la masa óptica para los registros trazables de cada día, usando el método de mínimos cuadrados, deberíamos poder ajustar la gráfica resultante a una recta cuya ordenada en el origen tomaría un valor muy próximo a \pm 0.005 y tendría una pendiente muy próxima a \pm 0.010 . Como, según lo explicado en el capítulo I, el valor de esta pendiente representa una estimación del error cometido en la calibración de los equipos implicados, tomaremos dicho valor como error de calibración diario, y substituyendo en (5) el valor de la masa óptica asociada a cada diferencia de AOD, estimaremos el error cometido debido al proceso de cálculo del AOD asociado a cada diferencia.

Este método presenta el inconveniente de que sólo podremos evaluar la influencia en la no trazabilidad del error de calibración, o del error en el proceso de cálculo del AOD, en aquellos días en los que disponemos, al menos, de dos diferencias de AOD trazables sobre las cuales aplicar el ajuste comentado. Por ello, en principio, tendremos que restringir el estudio a los días que cumplan con esta condición y nos permitan realizar tales ajustes. Por otro lado, cuantas más diferencias de AOD trazables tengamos para un mismo día, mejor será la estimación realizada sobre la influencia de cada tipo de error en la no trazabilidad. En la Tabla 2, se muestran los porcentajes de diferencias no trazables analizadas y no analizadas en cada canal. En la Figura 2, mostramos los resultados obtenidos para las diferencias no trazables analizadas.

\begin{tabular}{|c|cccc|}
\hline Canales (nm) & 380 & 440 & 500 & 870 \\
\hline Número de diferencias de AOD no trazables \\
{$[$ [IMEL - PFR]. IZO (2005 - 2015) } & 5680 & 3807 & 3916 & 2041 \\
Analizadas (\%) & 73.56 & 75.81 & 74.85 & 72.12 \\
No analizadas (\%) & 26.44 & 24.19 & 25.15 & 27.88 \\
\hline
\end{tabular}

Tabla 2: Diferencias de AOD no trazables y porcentajes analizados o no de las mismas, según el método de ajuste propuesto.

Para obtener las influencias individuales en la no trazabilidad de cada tipo de error general comentado anteriormente, así como su influencia conjunta mostradas en la Figura 2, hemos contabilizado, por un lado, el porcentaje de diferencias no trazables de AOD en las que el ajuste nos ha permitido obtener una ordenada en el origen cuyo valor absoluto es $\leq 0.005$, y al mismo tiempo, una pendiente en la recta cuyo valor absoluto es $>0.010$. Con ello, obtenemos la influencia en la no trazabilidad debida exclusivamente a errores superiores al $1 \%$ en la calibración; luego, contabilizamos el porcentaje de diferencias no trazables en las que el valor absoluto de la ordenada en el origen es $>0.005$ y el de la pendiente es $\leq 0.010$. Con ello, estimamos la influencia en la no trazabilidad debida exclusivamente al error en el cálculo del AOD asumiendo un error de calibración inferior o igual al I\%; finalmente, contabilizamos el porcentaje de diferencias no trazables con valor absoluto en la ordenada en el origen $>0.005 y$ con valor absoluto de la pendiente $>0.010$. Esto último, nos daría una estimación del efecto conjunto de los errores en el cálculo de AOD y por una calibración superior al $1 \%$. Todos estos porcentajes, han sido obtenidos respecto del número total de diferencias no trazables analizadas, que no son todas las que hay, sino todas aquellas en las que hemos podido ajustar mediante una recta las diferencias trazables de AOD diarias. 


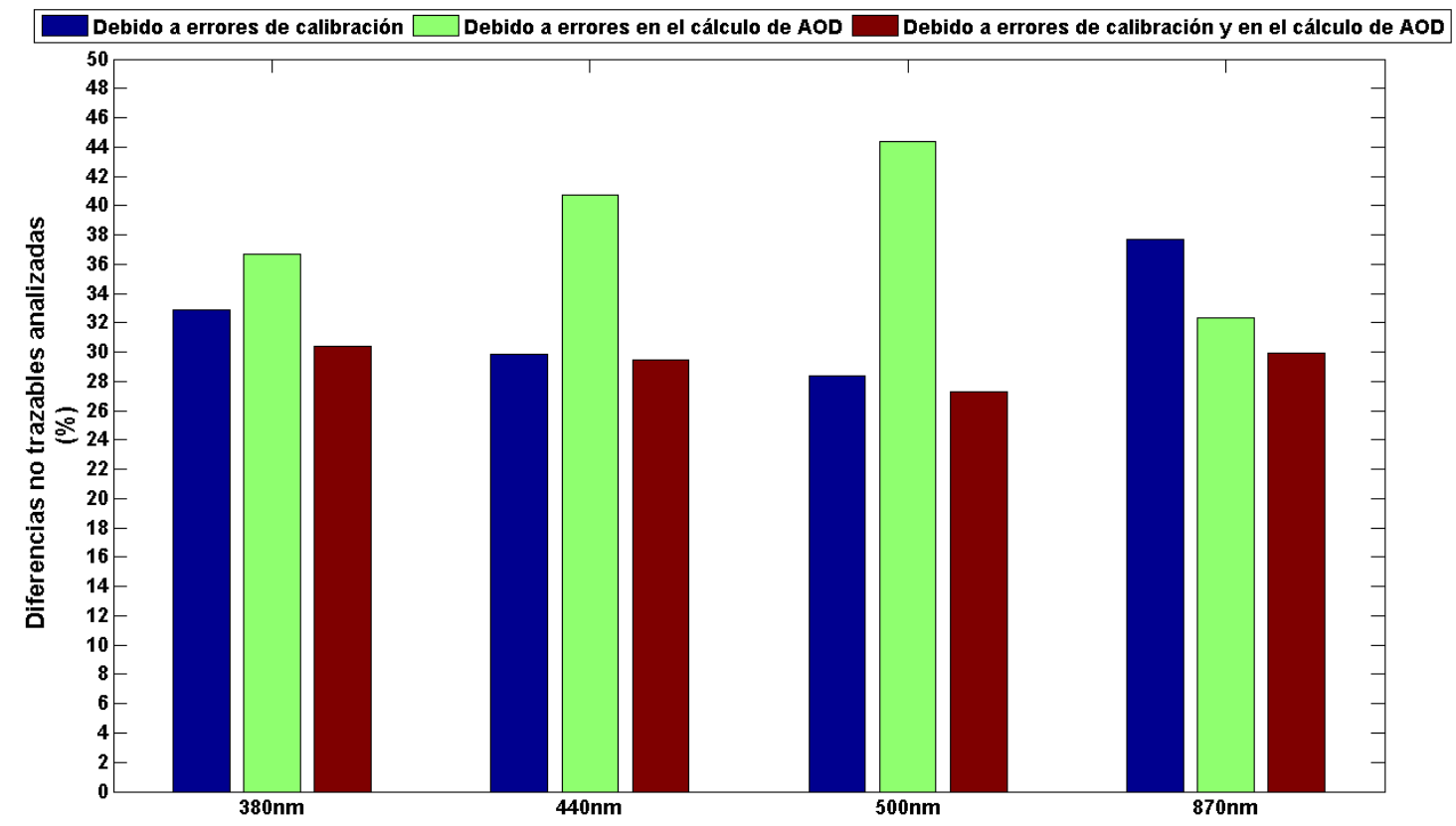

Figura 2: Influencia de los errores de calibración y del cálculo del AOD en las diferencias no trazables de AOD analizadas entre CIMEL y PFR, según canal. Los canales comparados del PFR se han extrapolado a las correspondientes longitudes de onda CIMEL usando los parámetros de Angstrom obtenidos por GAW-PFR. Las tres barras de cada grupo o canal suman el I00\% de las diferencias de AOD no trazables analizadas en dicho canal y corresponden a porcentajes cuya no trazabilidad se debe exclusivamente a la causa señalada en la leyenda. Observatorio de Izaña, 2005-20I5.

De la Tabla 2, podemos ver que se han analizado más del 70\% de las diferencias de AOD no trazables para cada uno de los canales aplicando el método descrito. Por otro lado, la Figura 2, nos muestra que para el canal de $870 \mathrm{~nm}$, la causa principal que conduce a la no trazabilidad en las diferencias de AOD son los errores de calibración. Para el resto de los canales, en cambio, son los errores en el cálculo del AOD la principal causa de la no trazabilidad en sus diferencias. Hay que señalar que estas estimaciones en los errores hay que atribuirlas a cualquiera de los dos equipos que se comparan en cada caso, o a los dos en conjunto, sin poder precisar más detalles. La razón por la que el canal de $870 \mathrm{~nm}$ sea el más afectado por los errores de calibración podría estar en la pequeña magnitud de los valores de AOD a esta longitud de onda. Estos valores son especialmente pequeños en los días en los que se calibra, ya que se exige que tales días sean muy estables y muy limpios (con un contenido muy bajo en aerosol, normalmente menor a 0.02 en torno a $500 \mathrm{~nm}$ ). De esta forma, un error ligeramente superior al $1 \%$ en la calibración de alguno de los equipos podría influir de forma especialmente sensible en los valores de AOD en este canal, y por tanto, en la trazabilidad.

A pesar del inconveniente comentado relativo a la restricción del número de diferencias de AOD no trazables analizadas debido al método propuesto, los tamaños de las muestras analizadas para cada canal, aseguran, con un nivel de confianza superior al $95 \%$ y un error muestral máximo del $2 \%$ en el canal de $870 \mathrm{~nm}$ y del $1 \%$ en el resto de los canales, la representatividad de dichas muestras respecto a la población total de diferencias de AOD no trazables en cada canal. Esto significa que, las conclusiones que vamos a sacar de aquí, son extrapolables con ese grado de confianza y de error, a toda la población de diferencias de AOD no trazables (y no sólo a las analizadas). En efecto, para que una muestra sea representativa de una determinada población, el tamaño adecuado de la muestra se relaciona con ciertos parámetros estadísticos según la relación siguiente:

$$
n=\frac{Z_{\alpha}^{2} \times N \times p \times q}{e^{2} \times(N-1)+Z_{\alpha}^{2} \times p \times q}
$$

Donde, $Z_{\alpha}$ es un estadístico que depende del nivel de confianza que asignemos según una distribución de dos colas normal y estándar (media 0 y desviación estándar I). Para un nivel de confianza del 95\%, toma el valor de $Z_{\alpha}=1.96$; para un nivel de confianza del $99 \% Z_{\alpha}=2.58$. Por otro lado, $e$ representa el máximo error muestral 
deseado en tanto por uno (\% dividido entre 100); $N$ es el número de datos o tamaño de la población total; $p$ es la proporción de datos de la población total que representa las características deseadas y $q=1-p$ es la proporción de datos de la población total que no representa las características deseadas. Cuando se desconocen estos dos últimos parámetros se suelen tomar ambos como $p=q=0.5$.

En la Tabla 3, se muestran los tamaños de muestras mínimos necesarios para la representatividad de la población total de diferencias de AOD no trazables, según el canal.

\begin{tabular}{|c|c|c|c|c|}
\hline $\begin{array}{c}\text { Canales (nm) } \\
\text { Tamaño población total no trazable } \\
\text { Error muestral (tanto por uno) }\end{array}$ & $\begin{array}{l}380 \\
N=5680 \\
e=0.01\end{array}$ & $\begin{aligned} & 440 \\
N & =3807 \\
e & =0.01\end{aligned}$ & $\begin{array}{l}500 \\
N=3916 \\
e=0.01\end{array}$ & $\begin{array}{c}870 \\
N=2041 \\
e=0.02\end{array}$ \\
\hline $\begin{array}{c}\text { Tamaño mínimo de muestra representativa } \\
\text { Tamaño de muestra real analizada }\end{array}$ & $\begin{array}{l}3569 \\
4178\end{array}$ & $\begin{array}{l}2727 \\
2886\end{array}$ & $\begin{array}{l}2782 \\
2931\end{array}$ & $\begin{array}{l}1103 \\
1472\end{array}$ \\
\hline
\end{tabular}

Tabla 3: Tamaño mínimo de muestra representativa de la población total de diferencias de AOD no trazables, según canal. Se ha obtenido a partir de la expresión (6) dada más arriba. El tamaño de muestra real corresponde al número de registros de cada canal con diferencias de AOD no trazables para las que se han podido estimar, por el procedimiento de ajuste lineal, la contribución de los diferentes errores a la no trazabilidad. En todos los casos, la muestra analizada supera en tamaño al mínimo necesario para su representatividad en la población total no trazable. Observatorio Atmosférico de Izaña $2005-20$ I 5.

\subsection{Influencia de variables de entrada en el $A O D$}

Por otro lado, debemos decir que el uso de diferentes bases de datos por parte de ambas redes para las variables de entrada en la fórmula de obtención del AOD influye en mayor o menor medida en la no trazabilidad de las diferencias de AOD.

En ambas redes, el cálculo del AOD se realiza a partir de la medida de irradiancia solar directa normal usando la expresión (2), que podemos aquí desarrollar de una forma más conveniente y detallada:

$$
\operatorname{AOD}(\lambda)=\frac{\ln \left(I_{0}(\lambda)\right)-\ln (I(\lambda))-2 \times \ln (S)-\sum_{i} \delta_{i}(\lambda) \times m_{i}}{m_{a}}
$$

donde $I_{0}(\lambda)$ es la señal extraterrestre o constante de calibración del fotómetro, $I(\lambda)$ es la señal de irradiancia solar directa normal medida por el fotómetro, $S$ es la distancia media Tierra-Sol del día en cuestión, expresada en Unidades Astronómicas (AU), $m_{a}$ es la masa óptica relativa del aerosol, $\delta_{i}(\lambda)$ son los coeficientes de extinción o atenuación (por absorción o dispersión) de la irradiancia directa a su paso a través de la atmósfera debida a la presencia de un cierto componente atmosférico $i$ y $m_{i}$ es la masa óptica relativa de dicho componente. Dependiendo de cuál sea la longitud de onda de trabajo, el componente atmosférico considerado y la cantidad de este componente presente en la columna atmosférica, la influencia del sumatorio en la expresión (7) será mayor o menor.

Los componentes atmosféricos principales distintos de los aerosoles que pueden afectar al cálculo del AOD, y por tanto, a la no trazabilidad de sus diferencias, y que se encuentran integrados dentro del sumatorio de la expresión (2) ó (7), son los siguientes: moléculas de aire, O3 (ozono) total en la columna atmosférica y NO2 (dióxido de nitrógeno) total en la columna atmosférica.

Para determinar cómo influye el uso de valores diferentes de presión, ozono o NO2 procedentes de bases de datos distintas para GAW-PFR y AERONET-CIMEL en el cálculo del AOD, el procedimiento a seguir sería reevaluar el AOD de los canales originales de una de las dos redes usando los valores de presión, ozono y NO2 que se han usado para el cálculo de AOD en la otra red, y luego, extrapolar los nuevos valores de AOD a las longitudes de onda exactas de esta última red. Sin embargo, si queremos cuantificar la influencia individual de cada una de las variables de entrada en el cálculo del AOD de los canales extrapolados y estimar el orden de magnitud correspondiente, nos resultará engorroso, ya que, el AOD de estos últimos, no lo hemos obtenido directamente de la fórmula de cálculo (7), sino a través de la ley de Angstrom usando los AOD de los canales originales. Por eso, nosotros seguiremos un procedimiento alternativo más sencillo. Demostraremos matemáticamente, que la variación 
en el $A O D$ para un cierto canal, ya sea extrapolado u original, debido a un determinado componente atmosférico, se puede poner en función de la variación correspondiente en el AOD de otro canal cualquiera que tomaremos como referencia (en nuestro caso, será el canal de 500nm del PFR). Esto, nos permitirá estudiar de una forma más sencilla la contribución de cada una de las variaciones en el AOD extrapolado a las diferencias no trazables entre AERONET-CIMEL y GAW-PFR y ver en qué medida pueden explicar la no trazabilidad.

\subsection{Influencia de la dispersión Rayleigh}

La extinción Rayleigh de luz directa por parte de las moléculas de aire debido a la dispersión es un efecto importante a tener en cuenta a la hora de evaluar el AOD y que debemos restar del valor de la extinción total de la irradiancia directa normal a su paso por la atmósfera. El valor de la extinción Rayleigh es mayor en los canales de menor longitud de onda. Las diferentes expresiones matemáticas que existen en la literatura y que se pueden usar para obtenerla pueden introducir pequeñas diferencias en el valor calculado. Una expresión sencilla alternativa al algoritmo de Bodhaine (Bodhaine et al., 1999) que se puede utilizar, tanto por parte de GAW-PFR, como por parte de AERONET-CIMEL para el coeficiente de extinción por dispersión Rayleigh, es la siguiente (Fröhlich C. and Shaw G. E., 1980):

$$
\delta_{R}(\lambda)=0.00864 \times \lambda^{-\left(3.916+0.074 \times \lambda+\frac{0.050}{\lambda}\right)} \times \frac{p}{1013.25}
$$

donde, $\lambda$ es la longitud de onda en micras $(\mu)$ y $p$ es la presión en $\mathrm{hPa}$ en el lugar de la medida. En el primer factor de esta expresión ya se incluye el factor de depolarización recomendado por Young (Young, 1980).

La extinción Rayleigh se calcula multiplicando el coeficiente de extinción Rayleigh dado por (8) por el cociente entre la masa óptica de Rayleigh y la masa óptica del aerosol. No se han utilizado exactamente los mismos valores de presión en el cálculo de esta componente de extinción por parte de ambas redes, ya que cada red utiliza su propio barotransmisor. En la Figura 3 (lzq.), se muestran los valores de presión usados por una y otra red y vemos que, en general, ambas series de presión siguen la misma variación y se encuentran dentro del mismo orden de magnitud. No obstante, hay valores puntuales de la presión medida por el barotransmisor de GAW-PFR que se alejan de la serie. De estos últimos, algunos de 2005 y 2006 no han sido representados en la Figura 3 (Izq.), ni su efecto en Figura 3 (Dcha.), para poder visualizar mejor los gráficos.
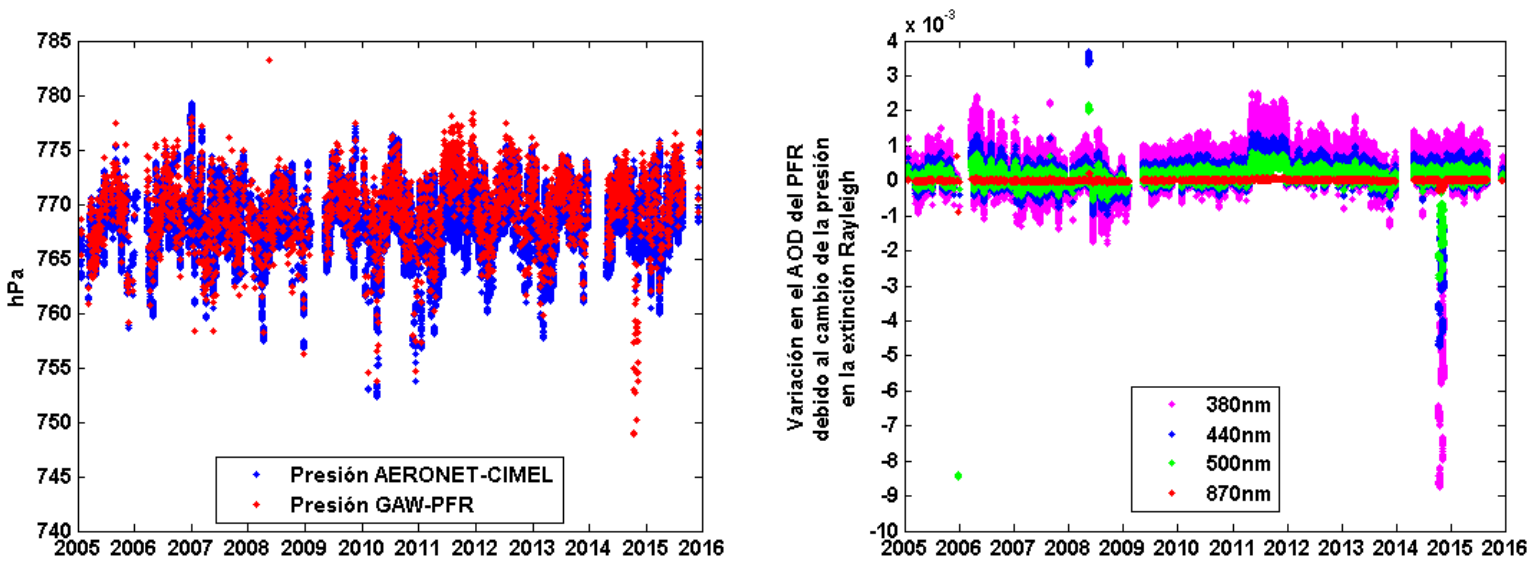

Figura 3: Izq.: Presiones usadas en Izaña por CIMEL y PFR para evaluar el AOD durante la comparación. Dcha.: Variación en el AOD del PFR para las diferentes longitudes de onda extrapoladas si hubiese usado el mismo valor de presión que usó CIMEL. Observatorio Atmosférico de Izaña, 2005-20 I 5.

Para estimar la influencia que tiene en el cálculo de AOD la extinción Rayleigh, sumaremos a los valores de AOD de los canales originales del PFR la extinción Rayleigh correspondiente a la longitud de onda de dichos canales originales obtenida usando los valores de presión del PFR, y luego, restaremos la extinción Rayleigh obtenida usando para el PFR los valores de presión que fueron usados por CIMEL. La diferencia entre el AOD original del PFR y el obtenido usando la presión CIMEL nos dará la estimación que buscamos en función de la longitud de onda del canal. Matemáticamente, el efecto neto de este proceso se puede expresar de la siguiente manera: 


$$
\begin{aligned}
& \Delta(A O D(\lambda))_{R}=\delta_{R}(\lambda)_{P_{P F R}} \times \frac{m_{R}}{m_{a}}-\delta_{R}(\lambda)_{P_{\text {CMEL }}} \times \frac{m_{R}}{m_{a}}= \\
& =\left[0.00864 \times \lambda^{-\left(3.916+0.074 \times \lambda+\frac{0.050}{\lambda}\right)} \times \frac{1}{1013.25} \times \frac{m_{R}}{m_{a}}\right] \times\left(P_{P F R}-P_{C I M E L}\right)
\end{aligned}
$$

En la expresión (9), $\Delta(A O D(\lambda))_{R}$ es la variación del AOD que se produce por un cambio en la extinción Rayleigh debido al uso de un valor diferente de presión. $P_{P F R}$ y $P_{C I M E L}$ son las presiones utilizadas por PFR y CIMEL en sus respectivos cálculos de AOD y $m_{R}$ y $m_{a}$ son las masas ópticas relativas de Rayleigh y del aerosol que vienen dadas en función de la elevación solar $\theta$ (expresada en grados), según las siguientes expresiones:

$$
\begin{gathered}
m_{R}=\frac{1}{\sin (\theta)+0.50572 \times(\theta+6.07995)^{-1.6364}} \quad \text { (10) } \quad \text { (Kasten and Young, 1989) } \\
m_{a} \approx m_{H 2 O}=\frac{1}{\sin (\theta)+0.0548 \times(\theta+2.65)^{-1.452}} \quad \text { (II) } \quad \text { (Kasten, 1966) }
\end{gathered}
$$

El aerosol se supone que es dominante en la troposfera y que tiene un perfil vertical de distribución similar al del contenido de vapor de agua, por eso, en GAW-PFR la masa óptica del aerosol se asimila a la del vapor de agua.

Ahora bien, la variación del AOD producido por un cambio en la extinción Rayleigh debido a un cambio en la presión para una determinada longitud de onda, puede ponerse en función de la correspondiente variación obtenida para otra longitud de onda a partir de (8). En efecto, para dos longitudes de onda diferentes $\lambda_{1}$ y $\lambda_{2}$, resulta que:

$$
\frac{\Delta\left(A O D\left(\lambda_{1}\right)\right)_{R}}{\Delta\left(A O D\left(\lambda_{2}\right)\right)_{R}}=\frac{\lambda_{1}^{-\left(3.916+0.074 \times \lambda_{1}+\frac{0.050}{\lambda_{1}}\right)}}{\lambda_{2}^{-\left(3.916+0.074 \times \lambda_{2}+\frac{0.050}{\lambda_{2}}\right)}}
$$

Esto es así, ya que las masas ópticas involucradas y el cambio en la presión son los mismos para una y otra longitud de onda. De esta forma, si conocemos cuál es la variación de AOD debido al cambio en la extinción Rayleigh en cualquiera de las longitudes de onda de los canales originales del PFR podremos estimar cuál ha sido la correspondiente variación en el AOD en cualquiera de las longitudes de onda extrapoladas del PFR sin más que aplicar la expresión (12). Tomando la variación del AOD en el canal de 500nm del PFR como referencia:

$$
\begin{aligned}
\Delta\left(A O D\left(\lambda_{1}=0.380\right)\right)_{R} & =3.1089 \times \Delta\left(A O D\left(\lambda_{2}=0.500\right)\right)_{R} \\
\Delta\left(A O D\left(\lambda_{1}=0.440\right)\right)_{R} & =1.6916 \times \Delta\left(A O D\left(\lambda_{2}=0.500\right)\right)_{R} \\
\Delta\left(A O D\left(\lambda_{1}=0.870\right)\right)_{R} & =0.1057 \times \Delta\left(A O D\left(\lambda_{2}=0.500\right)\right)_{R}
\end{aligned}
$$

En la Figura 3 (Dcha.) representamos estos resultados. Exceptuando las variaciones del AOD debidas a los valores puntuales de presión que estaban fuera de rango, la influencia de un cambio en la presión se traduce en una variación del AOD del PFR que es del orden de $10^{-4}$ para todos los canales excepto para el canal de $870 \mathrm{~nm}$ en el que es del orden de $10^{-5}$. Por tanto, se trata de una corrección muy pequeña. En este estudio hemos comprobado que un error en la presión de unos $20 \mathrm{hPa}$, puede ocasionar una variación en el $\mathrm{AOD}$ que puede ser como mucho de $9 \times 10^{-3}$. Los barotransmisores utilizados por los fotómetros tienen una precisión de $\sim 1 \mathrm{hPa}$, por lo que desviaciones de tal magnitud son sólo debido a mal funcionamiento de los barotransmisores instalados junto a los fotómetros. 


\subsubsection{Influencia de la absorción del ozono}

Se determina multiplicando el coeficiente de absorción de ozono $\sigma_{O_{3}}(\lambda)$, dependiente de la longitud de onda, por la cantidad de ozono $\mathrm{O}_{3}$ en la columna atmosférica:

$$
\delta_{O_{3}}(\lambda)=\sigma_{O_{3}}(\lambda) \times \frac{O_{3}(D U)}{1000}
$$

Esta última, suele medirse en unidades Dobson (DU) por instrumentos como los espectrofotómetros Brewer y Dobson o por satélites. El coeficiente de absorción de ozono suele venir dado en unidades de $\mathrm{cm}^{-1}$. Una unidad Dobson equivale a $1 / 1000 \mathrm{~cm}$, de ahí el factor de 1000 que aparece en (16). Cuando no se dispone del valor medido de ozono, se utiliza un valor climatológico. Por tanto, dependiendo del origen o fuente del dato de ozono puede haber diferencias en la extinción asociada al ozono que también deberá restarse de la extinción total a fin de obtener el espesor óptico de aerosoles.

La absorción de ozono interviene de forma más significativa en el canal de $500 \mathrm{~nm}$. Un valor típico, por defecto, del coeficiente de absorción de ozono para las longitudes de onda de $440 \mathrm{~nm}, 500 \mathrm{~nm}$ y $870 \mathrm{~nm}$, expresados en unidades de $\mathrm{cm}^{-1}$ (Gueymard, 1995), serían $\sigma_{O_{3}}(440 \mathrm{~nm})=0.00260, \sigma_{O_{3}}(500 \mathrm{~nm})=0.03150$ y $\sigma_{O_{3}}(870 \mathrm{~nm})=0.00133$. Para los canales de $440 \mathrm{~nm}$ y $870 \mathrm{~nm}$, los coeficientes son ya muy pequeños, especialmente para este último canal. Por otro lado, el canal de $380 \mathrm{~nm}$ no presenta absorción por ozono.

La extinción por ozono se calcula multiplicando la expresión (16) por la masa óptica del ozono y dividiendo por la masa óptica del aerosol. Entonces, para estimar la variación de AOD en el PFR debido a un cambio en el valor de ozono, procedemos de forma análoga a como hicimos con la extinción Rayleigh: primero sumamos al AOD del PFR la extinción de ozono calculada usando el valor de ozono PFR, y luego, le restamos la extinción de ozono obtenida usando el mismo valor de ozono que usó CIMEL.

GAW-PFR usa, para la masa óptica del ozono, la expresión siguiente (Komhyr, 1980):

$$
m_{O_{3}}=\frac{R+h}{\sqrt{(R+h)^{2}-(R+r)^{2} \times \cos ^{2}(\theta)}}
$$

donde $R=6370 \mathrm{~km}$ es el radio medio de la Tierra, $r=2.370 \mathrm{~km}$ es la altitud de la estación, $h=22 \mathrm{~km}$ es la altura estimada de la capa de ozono y $\theta\left({ }^{\circ}\right)$ es la elevación solar. Sin embargo, AERONET-CIMEL usa una expresión similar pero en versión más actualizada (Komhyr et al., 1989) que no supone un valor fijo de altura de la capa de ozono de $22 \mathrm{Km}$ sino que el valor se ajusta en función de la latitud del lugar.

La variación neta en el AOD del PFR, al usar el valor de ozono CIMEL, puede estimarse de la siguiente manera:

$$
\begin{aligned}
& \Delta(A O D(\lambda))_{O_{3}}=\delta_{O_{3}}(\lambda)_{P F R}-\delta_{O_{3}}(\lambda)_{\text {CIMEL }}= \\
& =\sigma_{O_{3}}(\lambda) \times \frac{O_{3(P F R)}}{1000} \times \frac{m_{O_{3}}}{m_{a}}-\sigma_{O_{3}}(\lambda) \times \frac{O_{3(C I M E L)}}{1000} \times \frac{m_{O_{3}}}{m_{a}}= \\
& =\sigma_{O_{3}}(\lambda) \times \frac{1}{1000} \times \frac{m_{O_{3}}}{m_{a}} \times\left(O_{3(P F R)}-O_{3(\text { CIMEL })}\right)
\end{aligned}
$$

Utilizando la expresión (18) para dos longitudes de onda distintas, se puede poner la variación en el AOD en una de ellas en función de la de la otra de forma análoga a como hicimos en el apartado anterior. De esta forma, podemos estimar cuál es dicha variación de AOD en las longitudes de onda extrapoladas conocida dicha variación en alguna de las longitudes de onda originales del PFR. 


$$
\frac{\Delta\left(A O D\left(\lambda_{1}\right)\right)_{O_{3}}}{\Delta\left(A O D\left(\lambda_{2}\right)\right)_{O_{3}}}=\frac{\sigma_{O_{3}}\left(\lambda_{1}\right)}{\sigma_{O_{3}}\left(\lambda_{2}\right)}
$$

Tomando como referencia la variación del AOD por ozono en 500nm del PFR, resulta que:

$$
\begin{aligned}
& \Delta\left(A O D\left(\lambda_{1}=0.440\right)\right)_{O_{3}}=0.0825 \times \Delta\left(A O D\left(\lambda_{2}=0.500\right)\right)_{O_{3}} \\
& \Delta\left(A O D\left(\lambda_{1}=0.870\right)\right)_{O_{3}}=0.0422 \times \Delta\left(A O D\left(\lambda_{2}=0.500\right)\right)_{O_{3}}
\end{aligned}
$$

En la Figura 4 (Izq.) se muestran los valores de ozono utilizados por PFR y CIMEL, y en la Figura 4 (Dcha.), se muestran las estimaciones en las variaciones netas del AOD por cambio en la cantidad de ozono relacionadas con las longitudes de onda extrapoladas.
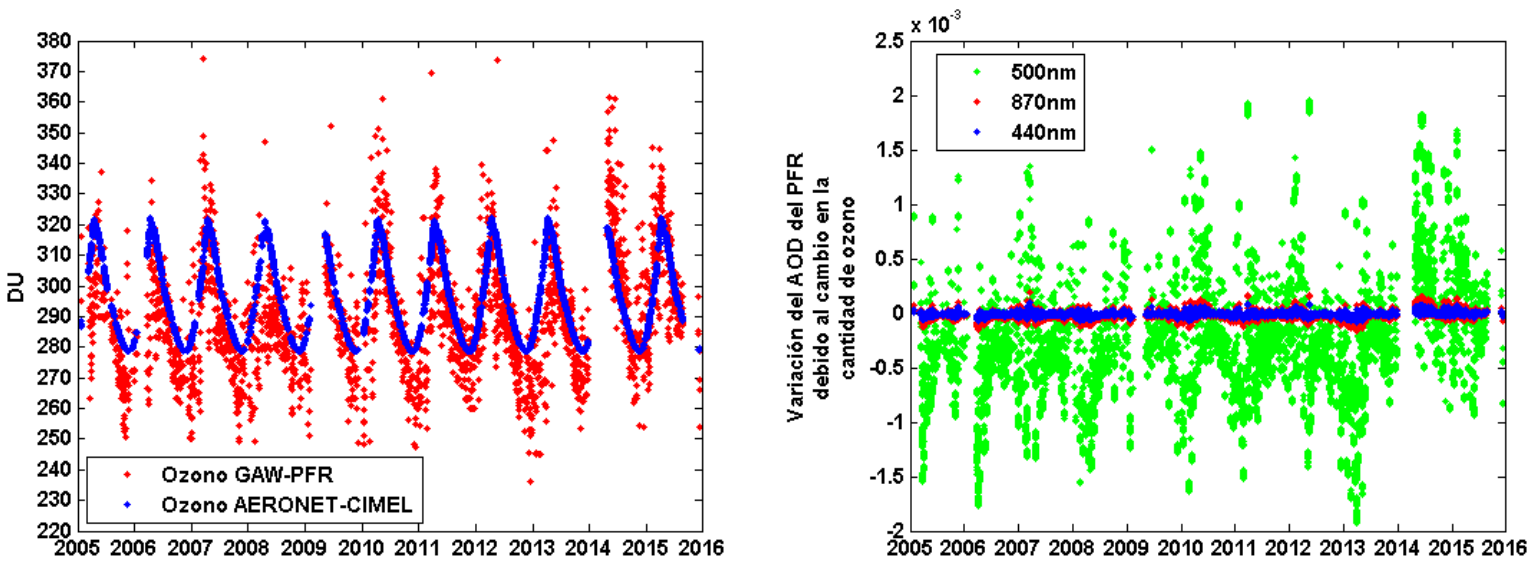

Figura 4: Izq.: Valores de ozono usados en Izaña por CIMEL y PFR para evaluar el AOD durante la comparación. Dcha.: Variación en el AOD del PFR para las diferentes longitudes de onda extrapoladas si el AOD PFR se hubiese calculado usando el mismo valor de ozono que usó CIMEL. Observatorio Atmosférico de Izaña, 2005-20 I5.

La gran mayoría de las variaciones de AOD debidas al ozono para el canal de $500 \mathrm{~nm}$, que es en el que se presenta el mayor coeficiente de absorción de ozono, son del orden de $10^{-4}$ mientras que, para los canales de $440 \mathrm{~nm}$ y $870 \mathrm{~nm}$, estas variaciones están en el orden de $10^{-5} \mathrm{e}$ incluso, $10^{-6}$. Por tanto, su influencia en las diferencias de AOD y en la no trazabilidad de estos será despreciable para nuestra estación. Debe advertirse que la variación estacional del ozono en la región subtropical es muy pequeña (en torno a $50 \mathrm{DU}$ ) pero en latitudes altas pueden registrarse variaciones de ozono superiores a $100 \mathrm{DU}$, por lo que el canal de $500 \mathrm{~nm}$ sí podría verse afectado por la absorción del ozono.

\subsubsection{Influencia de la absorción del NO2}

La absorción de este gas puede llegar a ser importante por debajo de los $700 \mathrm{~nm}$. También se obtiene como producto de un coeficiente de absorción (que depende de la longitud de onda y viene expresado normalmente en $\mathrm{cm}^{-1}$ ), por la cantidad de NO2 en columna, la cual, viene expresada habitualmente en Unidades Dobson (DU). Al igual que con el ozono, dependiendo del origen o fuente del dato de NO2, puede haber discrepancias en el valor de su extinción asociada, y por tanto, también en el AOD.

La extinción debida al NO2 se calcula multiplicando su coeficiente de absorción por el cociente entre la masa óptica del NO2 y la masa óptica del aerosol.

Para calcular esta extinción, usaremos como aproximación para la masa óptica del NO2, la siguiente expresión (Gueymard, 1995): 


$$
m_{N O_{2}}=\frac{1}{\sin (\theta)+602.30 \times(90-\theta)^{0.5} \times(27.96+\theta)^{-3.4536}}
$$

En la metodología utilizada por GAW-PFR para el cálculo del AOD, no se tiene en cuenta la absorción de este gas en ninguno de los canales originales, mientras que en la metodología AERONET-CIMEL sí se tiene en cuenta. La variación neta en el AOD de los canales originales del PFR, si se hubiese tenido en cuenta la absorción de NO2, vendría dada por:

$$
\Delta(A O D(\lambda))_{N O 2}=-\delta_{N O 2}(\lambda) \times \frac{m_{N O 2}}{m_{a}}=-\sigma_{N O 2}(\lambda) \times \frac{N O_{2(C I M E L)}}{1000} \times \frac{m_{N O 2}}{m_{a}}
$$

Donde $\sigma_{N O 2}(\lambda)$ es el coeficiente de absorción del NO2, $N O_{2(C I M E L)}$ es la cantidad de dióxido de nitrógeno en la columna atmosférica expresada en DU empleada por CIMEL y $m_{\mathrm{NO} 2}$ y $m_{a}$ son las masas ópticas relativas de NO2 y aerosol dadas respectivamente por (22) y (II).

Los AOD originales del PFR tendrán que ser minimizados en la cantidad dada por (23), según la longitud de onda del canal considerado.

Y, análogamente, para dos longitudes de onda diferentes:

$$
\frac{\Delta\left(A O D\left(\lambda_{1}\right)\right)_{N O 2}}{\Delta\left(A O D\left(\lambda_{2}\right)\right)_{N O 2}}=\frac{\sigma_{N O 2}\left(\lambda_{1}\right)}{\sigma_{N O 2}\left(\lambda_{2}\right)}
$$

Teniendo en cuenta, los valores de los coeficientes de absorción del NO2 para las diferentes longitudes de onda (Gueymard, 1995), tenemos que la variación del AOD en los canales extrapolados del PFR como consecuencia de la absorción del NO2, tomando como referencia la del canal original de $500 \mathrm{~nm}$ del PFR, vendrá dada por:

$$
\begin{aligned}
& \Delta\left(A O D\left(\lambda_{1}=0.380\right)\right)_{N O 2}=3.3766 \times \Delta\left(A O D\left(\lambda_{2}=0.500\right)\right)_{N O 2} \\
& \Delta\left(A O D\left(\lambda_{1}=0.440\right)\right)_{N O 2}=2.6623 \times \Delta\left(A O D\left(\lambda_{2}=0.500\right)\right)_{N O 2}
\end{aligned}
$$

En el canal de $870 \mathrm{~nm}$ no se produce absorción por $\mathrm{NO} 2$ con lo que la variación del AOD en este canal, debido a este gas, es nula.

En la Figura 5 (Izq.) representamos la cantidad de NO2 utilizada por CIMEL para evaluar el AOD. En la Figura 5 (Dcha.) se muestra la variación producida en el AOD de los canales extrapolados del PFR como consecuencia de considerar la absorción por NO2. La variación del AOD por causa del NO2 es del orden de $10^{-3}$ para los canales de $380 \mathrm{~nm}$ y $440 \mathrm{~nm}$, mientras que, para el canal de $500 \mathrm{~nm}$, es del orden de $10^{-4}$. Al igual que para el ozono, la absorción debida al NO2 en columna es despreciable en la determinación del AOD en nuestra región subtropical. Sin embargo, esta puede suponer un efecto importante en la determinación de AOD en aquellas regiones muy afectadas por contaminación atmosférica y en las cuales puedan registrarse valores de NO2 en columna muy superiores a los utilizados y procedentes de una climatología (ver, por ejemplo, Chubarova et al., 2016). 

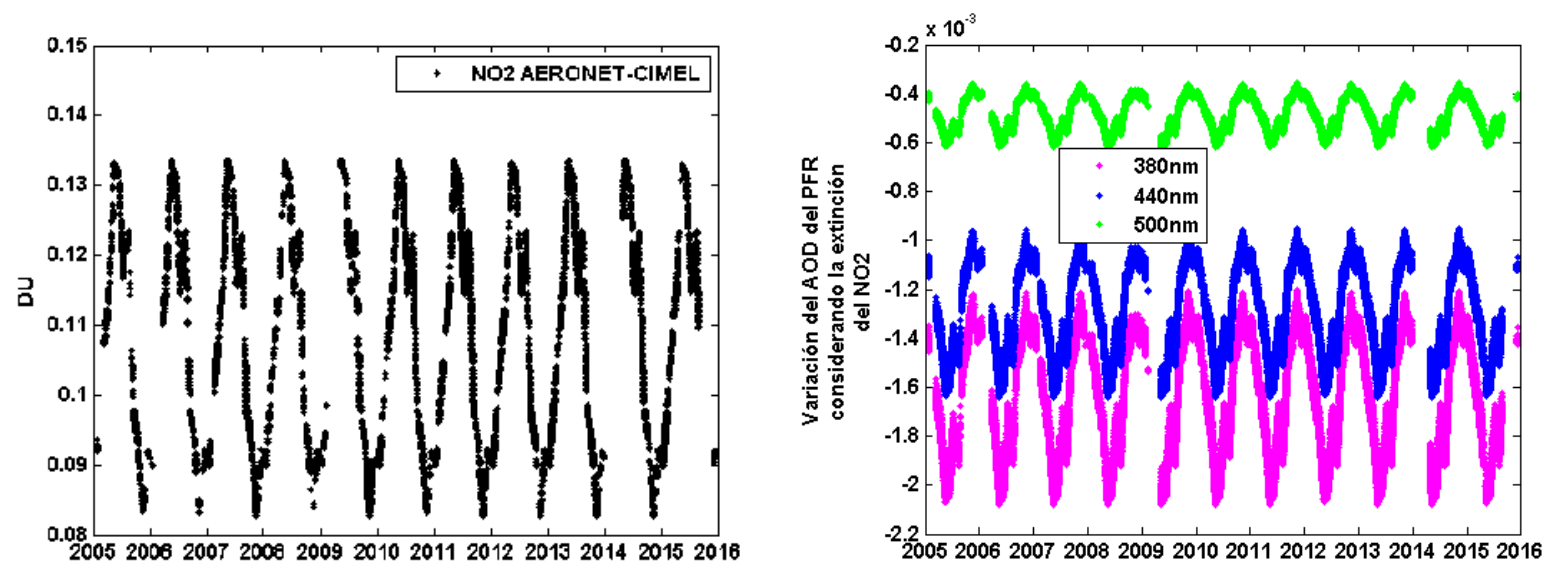

Figura 5: Izq.: Valores de NO2 usados en Izaña por CIMEL para evaluar el AOD durante la comparación. Dcha.: Variación en el AOD del PFR para las diferentes longitudes de onda extrapoladas si se hubiese tenido en cuenta la absorción del NO2 en el cálculo del AOD. Observatorio Atmosférico de Izaña, 2005-20 I 5.

\subsubsection{Efecto de la combinación de componentes}

Lo estudiado hasta ahora es la contribución por separado de los diferentes parámetros de entrada en el cálculo del AOD a la variación del AOD extrapolado del PFR. Sin embargo, la contribución conjunta de todos ellos no será la suma de las contribuciones de cada uno por separado sino que se producirán cancelaciones parciales de algunas de estas variaciones debido a su compensación por otras. Tenemos que ver en qué medida estas variaciones individuales y conjuntas pueden explicar los casos de no trazabilidad detectados.

En la Figura 6 (Izq.), representamos la variación total o conjunta del AOD del PFR si lo hubiésemos evaluado usando simultáneamente los datos de presión, ozono y NO2 que han sido utilizados por CIMEL. En la Figura 6 (Dcha.), representamos los percentiles 5, 25, 50, 75 y 95 de esta variación conjunta del AOD, según el canal.
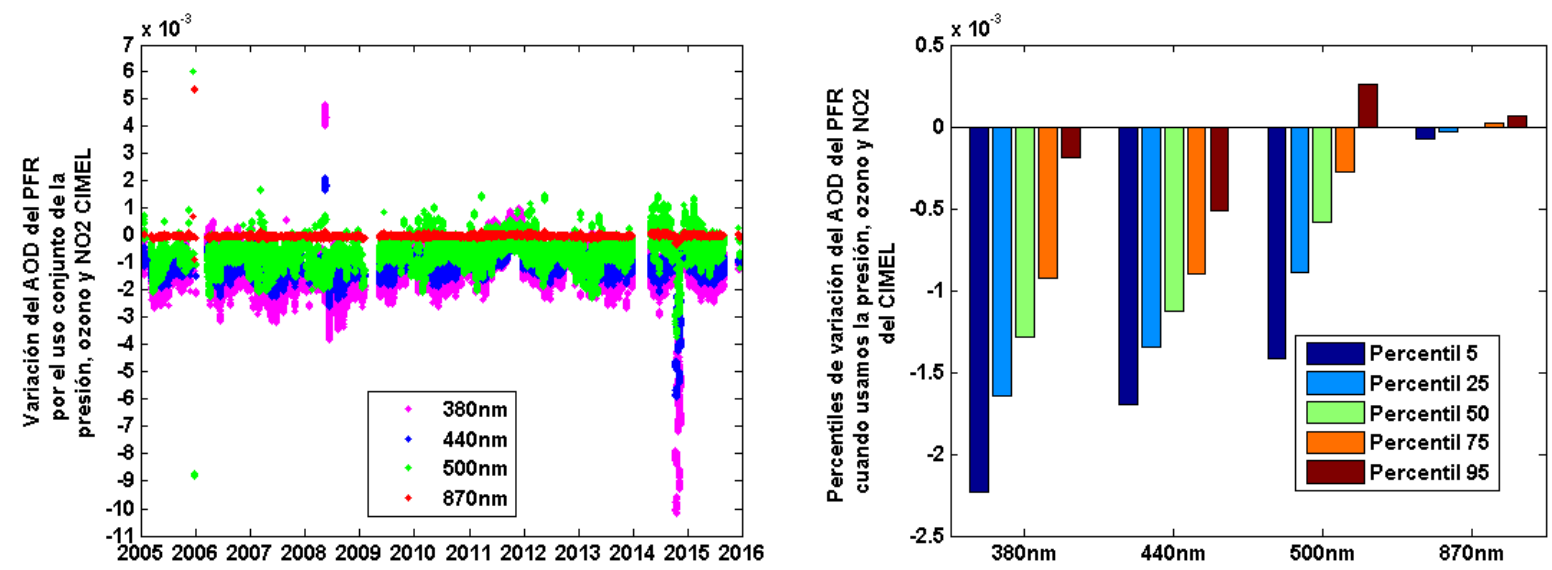

Figura 6: Izq.: Variación del AOD del PFR como consecuencia de usar, simultáneamente, los valores de presión, ozono y NO2 usados por CIMEL en cada canal. Dcha.: Percentiles de la variación del AOD para las diferentes longitudes de onda extrapoladas. Observatorio Atmosférico de Izaña, 2005-20I5.

De esta manera, estimamos que el efecto conjunto de un cambio en los parámetros meteorológicos de entrada en el cálculo del AOD debido al uso de bases de datos distintas para la presión, ozono y NO2, se traduce en una disminución generalizada de las diferencias de AOD del orden de entre $10^{-4}$ y $10^{-3}$ para todos los canales, excepto para el de $870 \mathrm{~nm}$, en el que el cambio sería del orden de $10^{-5}$. Por tanto, despreciable. 
En la Figura 7, se muestra qué porcentaje de diferencias inicialmente no trazables se han "trazabilizado" como consecuencia de haber realizado una corrección en el AOD del PFR debida a cualquiera de los componentes atmosféricos aquí analizados o a cualquier combinación de los tres.

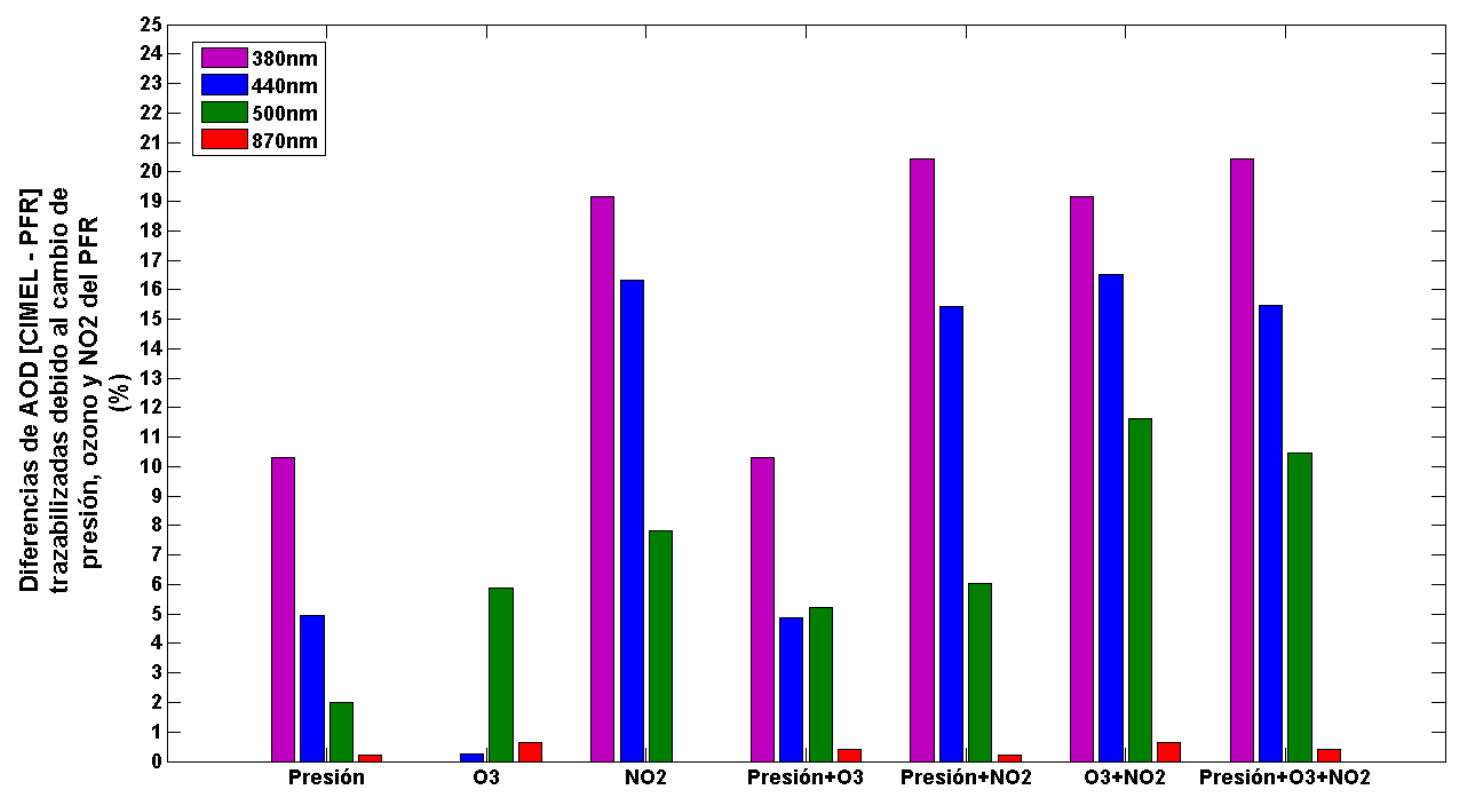

Figura 7: Porcentaje de diferencias de AOD CIMEL - PFR no trazables que se han "trazabilizado" como consecuencia de haber reevaluado el AOD del PFR asumiendo los mismos valores de presión, O3 y NO2 que usó CIMEL en su evaluación de AOD. La gráfica muestra el porcentaje de diferencias trazabilizadas asumiendo para el AOD del PFR únicamente la variación en uno de los componentes atmosféricos, en los tres, o en la combinación de dos cualesquiera de ellos. Todos los porcentajes se calculan respecto del número total de diferencias no trazables en cada canal. Observatorio Atmosférico de Izaña, 2005-20I 5.

Como puede verse en la gráfica, el aumento en la trazabilidad debida al cambio del O3 y del NO2 en los canales de $380 \mathrm{~nm}$ y $870 \mathrm{~nm}$, respectivamente, es nula ya que dichos componentes no presentan absorción en los respectivos canales.

También se observa que, para el canal de $380 \mathrm{~nm}$, el uso del valor de presión CIMEL combinado con la contribución que aportaría considerar la extinción causada por el NO2 en dicha longitud de onda, supone hasta un $20 \%$ de aumento de la trazabilidad (o de disminución de la no trazabilidad) del AOD del PFR con respecto al CIMEL en los datos no trazables analizados que representan un $73.56 \%$ del total. En el caso de los canales de $440 \mathrm{~nm}$ y de $500 \mathrm{~nm}$, la mayor influencia en la mejora de la trazabilidad se da al tener en cuenta el efecto conjunto de la absorción del NO2 y del valor del ozono CIMEL en dichos canales. Con ello, en 440nm, conseguimos aumentar la trazabilidad (o disminuir la no trazabilidad) en algo más del $16 \%$ de los datos no trazables analizados (que son un $75.81 \%$ del total), y en el caso de $500 \mathrm{~nm}$, en algo más del II\%. Finalmente, para el canal de $870 \mathrm{~nm}$, la consideración del ozono CIMEL nos permite aumentar sólo en un $0.64 \%$, aproximadamente, la trazabilidad entre ambas redes. Podemos decir que, en este último canal, no tiene influencia en la trazabilidad del AOD la diferencia de bases de datos en los parámetros de entrada de cálculo del AOD. Todos estos porcentajes están referidos respecto del número de diferencias no trazables de cada canal.

En la Tabla 4, mostramos el efecto que tiene en la trazabilidad total (porcentajes calculados respecto del número total de diferencias disponibles totales en cada canal) la reevaluación del AOD del PFR con los mismos parámetros de entrada de presión, ozono y NO2 que usó CIMEL.

Los números de la tabla que están en azul, muestran los valores máximos alcanzados en la trazabilidad de cada canal asociados a la modificación del componente o componentes que aparecen en la fila de la izquierda. 


\begin{tabular}{cccccc}
\hline $\begin{array}{c}\text { Trazabilidad AOD [CIMEL-PFR] } \\
\text { Izaña (2005-20I5) }\end{array}$ & $\mathbf{3 8 0}$ & $\mathbf{4 4 0}$ & $\mathbf{5 0 0}$ & $\mathbf{8 7 0}$ \\
\hline \multicolumn{2}{c}{ Original (\%) } & 91.67 & $\mathbf{9 4 . 4 9}$ & $\mathbf{9 4 . 2 6}$ & $\mathbf{9 7 . 0 4}$ \\
Modificada & Rayleigh & 91.97 & 94.44 & 94.22 & 96.99 \\
(\%) por & O3 & 91.67 & 94.49 & 94.40 & 97.04 \\
corrección & NO2 & 92.08 & 94.64 & 94.47 & 97.04 \\
en AOD & Rayleigh+O3 & 91.97 & 94.44 & 94.37 & 96.98 \\
PFR & Rayleigh+NO2 & $\mathbf{9 2 . 6 6}$ & $\mathbf{9 4 . 7 0}$ & 94.46 & 96.99 \\
debida a: & O3+NO2 & 92.08 & 94.63 & 94.57 & 97.04 \\
& Rayleigh+O3+NO2 & 92.66 & 94.70 & $\mathbf{9 4 . 5 9}$ & 96.98
\end{tabular}

Tabla 4: Trazabilidad original y modificada reevaluando el AOD del PFR asumiendo para el cálculo los mismos parámetros de entrada que CIMEL, según se especifica en la fila correspondiente. Los porcentajes se han calculado con respecto al número total de diferencias disponibles en cada canal. En rojo, valores de trazabilidad original que se han podido mejorar; en azul, valores de trazabilidad más altos conseguidos. Observatorio Atmosférico de Izaña, 2005-20I5.

De esta forma, se observa que los aumentos respectivos del $0.99 \%$ y del $0.21 \%$ en la trazabilidad total de los canales de $380 \mathrm{~nm}$ y de $440 \mathrm{~nm}$ se deben al cambio del valor de presión y a la consideración de la extinción por NO2, ya que el ozono tiene una influencia nula o despreciable en tales canales. Para el canal de 500nm, el mayor aumento de trazabilidad es de un $\mathbf{0 . 3 3} \%$ que se consigue teniendo en cuenta la contribución de los tres componentes, ozono, NO2 y presión, si bien, la influencia de esta última es muy pequeña y sólo mejora la trazabilidad cuando está combinada con cualquiera de los otros dos componentes. Finalmente, en el canal de 870nm, no se aprecia ninguna mejora sobre la trazabilidad original debido al cambio de cualquiera de estos componentes o de sus combinaciones.

Hay que observar que para obtener los porcentajes de trazabilidad expresados en la Tabla 4 , se han aplicado los cambios de presión, ozono y NO2 a las series completas de AOD de cada uno de los canales, es decir, tanto a las diferencias trazables como no trazables. Por ello, estos porcentajes están referidos al número total de diferencias disponibles en cada canal. Estos cambios de presión, ozono y NO2 aplicados a todas las diferencias de AOD dan lugar también a que algunas de estas diferencias, inicialmente trazables, se hayan convertido en no trazables. Pero el efecto neto, es de una mejora en la trazabilidad total en todos los canales por debajo de $870 \mathrm{~nm}$. El aplicar los cambios a todas las diferencias, trazables y no trazables, también explica que los aumentos en los porcentajes de la Tabla4 no se correspondan exactamente con el componente o combinación de componentes para los que se producían los aumentos en la trazabilidad cuando los cambios se aplicaban únicamente a las diferencias no trazables de la Figura 7.

\subsection{Influencia de la nubosidad}

Finalmente, nos faltaría considerar el efecto que tiene en la no trazabilidad los posibles fallos en el algoritmo de detección de nubes. Este algoritmo, empleado por una y otra red con ciertas variantes, supone que el paso de una nube es algo transitorio, relativamente rápido y que provoca un cambio brusco y apreciable en el $A O D$. Este cambio brusco en el AOD debido a la nube se detectaría midiendo la estabilidad de tripletes de medidas, es decir, de paquetes de tres medidas de intensidad, muy próximas entre sí, de forma que, cuando pasa una nube la desviación estándar respecto de la media de los tripletes se dispara por encima de cierto valor. Sin embargo, esto no siempre es así. No ocurriría, por ejemplo, cuando la nube es estratiforme y de gran extensión horizontal y/ó cuando es delgada como un cirro tenue. En el primer caso, la estabilidad del triplete se mantendría dentro de la nube y el algoritmo la consideraría como una situación de background similar a la que pudiera darse en situación de calima o polvo persistente. En el segundo caso, al ser una nube muy delgada la fluctuación en el AOD sería pequeña y no sería posible discernir si se trata del efecto de la nube delgada, o de ruido en la señal, o de presencia débil de polvo. En estos casos, los algoritmos de detección de nubes ("cloudscreening") pueden fallar de forma dispar en las redes GAW-PFR y AEONET-CIMEL.

Estos casos podrían estar presentes en el fichero de registros cruzados entre CIMEL y PFR al no ser detectados por los algoritmos de filtrado. Esto es posible porque, aunque el cruce entre los AOD de una y otra red se haya realizado con datos coincidentes en el minuto, los equipos no están enfocando exactamente al mismo lugar del cielo en dicho minuto y un cirro delgado, por ejemplo, podría entrar parcialmente en el campo visual de alguno de los dos fotómetros y no en el otro; incluso enfocando al mismo lugar del cielo, también podría darse este hecho debido a que ambos tipos de fotómetros poseen diferentes campos de visión angular (FOV, ver Tabla I). 
Detectar la posible presencia de este tipo de registros es muy complejo, dada la casuística tan variada de situaciones, y no tendríamos la certeza al 100\% de obtener los resultados esperados puesto que habría que estudiar, por un lado, los días con presencia de nubes estratiformes y estables con diferentes grosores, y por otro, los días despejados o casi despejados con presencia espúrea de nubes delgadas. Además, ambas situaciones deberían estudiarse en casos de presencia o no de polvo. Sin embargo, en cualquiera de estos casos, aunque la trazabilidad no se cumpla, el $A O D$ de uno y otro equipo será parecido, y su diferencia, relativamente pequeña. $\mathrm{Si}$, por el contrario, lo que se observa es una gran diferencia entre ambos AOD, entonces hay que descartar a priori, que la causa sea la presencia de una nube no detectada. En estos casos, habría que barajar la hipótesis de que se tratara de un problema en la señal de alguno de los dos equipos como consecuencia de un ruido excesivo, un fallo en el apuntamiento, una disminución drástica de intensidad en la señal debida a algún obstáculo no nuboso que se interpone, o alguna avería o fallo en el sistema.

No disponemos de información suficiente y solapada con el período de estudio para poder evaluar en detalle la influencia de las nubes en la no trazabilidad del AOD. Tampoco tenemos forma de discernir cuáles de las diferencias de AOD que son lo suficientemente grandes como para no cumplir con los criterios de trazabilidad, corresponden, al mismo tiempo, a valores lo suficientemente pequeños de AOD como para pasar los filtros de detección de nubes de los algoritmos de ambas redes; y de estos valores, cuáles se deben realmente a nubes persistentes y estables o simplemente a carga de aerosoles en cantidades variables. Así que, estudiaremos de una forma muy genérica la no trazabilidad en aquellos días en los que hay presencia de nubes (independientemente de que hayan cruzado o no el campo visual de los fotómetros implicados) frente a aquellos otros que son completamente despejados. Para ello, se han seleccionado, por inspección visual, aquellos días en el período 20052015 en los que no se detecta traza alguna de nubes. Esto se ha hecho observando la curva de radiancia global diaria desde 2005 a 2008 obtenida a partir de equipos del Centro Radiométrico Nacional (CRN), y desde 2009 a 2015, a partir de equipos de la Baseline Surface Radiation Network (BSRN). Todos ellos, midieron de forma simultánea con los fotómetros CIMEL y PFR y se hallan ubicados en el mismo lugar que aquellos, o sea, en la Torre de Medidas del Observatorio Atmosférico de Izaña.

Para determinar la máxima influencia asociada a la nubosidad en la no trazabilidad cruzamos los registros de las diferencias de $A O D$ en cada canal con los días despejados, por un lado, y no despejados, por otro, y seleccionamos aquellas diferencias de AOD no trazables donde los errores de calibración se mantienen, en valor absoluto, por debajo del $1 \%$. Con esta selección, descartamos como causa de no trazabilidad una calibración deficiente. Los resultados obtenidos son los siguientes:

Para el canal de $380 \mathrm{~nm}$, el $33.83 \%$ de estas diferencias de AOD seleccionadas se distribuyen en días despejados, y el $66.17 \%$ restante se distribuyen en días en los que hay presencia de nubes. Entonces, en primera aproximación, estimamos que en el canal de $380 \mathrm{~nm}$ la presencia de nubosidad tiene una influencia máxima sobre la no trazabilidad del $32.34 \%$ que sería la diferencia entre los dos porcentajes anteriores. En $440 \mathrm{~nm}$, la no trazabilidad en días despejados es del $33.87 \%$ y para días con presencia de nubes es del $66.13 \%$. Así que la influencia máxima de las nubes en la no trazabilidad del AOD en este canal se estima en un $32.26 \%$. Para $500 \mathrm{~nm}$, tenemos un $33.82 \%$ en días despejados y un $\mathbf{6 6 . 1 8 \%}$ para días con nubes, por lo que la estimación de la influencia máxima nubosa en la no trazabilidad sería en este caso del $32.36 \%$. Finalmente, en $870 \mathrm{~nm}$, el porcentaje de diferencias de AOD no trazables es del $48.74 \%$ en días despejados y del $51.26 \%$ en días no despejados. Esto supone una influencia máxima de la nubosidad del $2.52 \%$ en la no trazabilidad del AOD en este canal. Todos estos porcentajes se han calculado contabilizando el número de diferencias no trazables con errores de calibración inferiores al I\% que corresponden a días despejados por un lado, y no despejados por otro, y referidas sobre el total de diferencias no trazables de cada canal (días despejados y no despejados) en las que el error de calibración es inferior al $1 \%$.

Para profundizar un poco más sobre la influencia generalizada de la presencia de nubes en la no trazabilidad del AOD, hemos relacionado las diferencias de AOD trazables y no trazables con la denominada fracción diaria de cielo despejado (FCS). Este factor se define como el porcentaje de horas de insolación observadas respecto del número máximo de horas de insolación diaria posible (García et al., 2014). Cuanto más alto sea el FCS diario mayor porcentaje de cielo despejado tenemos en ese día. En la Figura 8, representamos para cada canal los porcentajes de diferencias de AOD trazables y no trazables contenidas en cada intervalo FCS. 

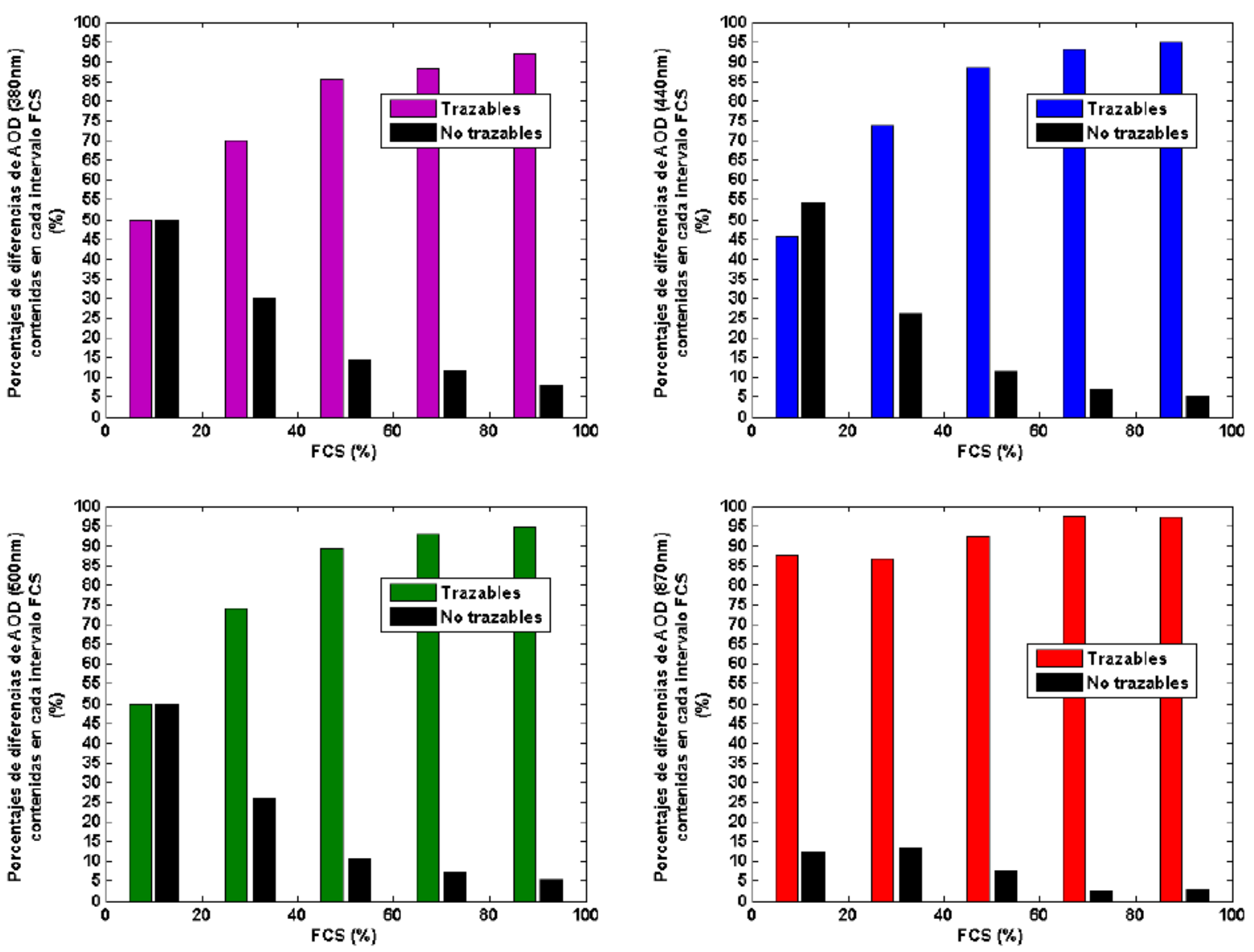

Figura 8: Influencia de la cantidad de nubes presentes en el cielo en el número de diferencias de AOD [CIMEL - PFR] trazables y no trazables de cada canal. De izquierda a derecha, y de arriba abajo, se muestran los resultados de los canales de $380 \mathrm{~nm}$, $440 \mathrm{~nm}, 500 \mathrm{~nm}$ y $870 \mathrm{~nm}$. Los porcentajes calculados en cada intervalo FCS están referidos respecto del número total de diferencias de AOD disponibles en ese intervalo. Observatorio Atmosférico de Izaña, 2005 - 20 I5.

La Figura 8 nos muestra que, por debajo de un FCS del 20\%, y para longitudes de onda inferiores a $870 \mathrm{~nm}$, la no trazabilidad de las diferencias de AOD [CIMEL - PFR] supone al menos un $50 \%$ del total de diferencias contenidas en dicho intervalo. Esto significa que la presencia de nubes posee una influencia significativa en la no trazabilidad del AOD para longitudes de onda en el rango visible. A medida que la fracción de cielo despejado va en aumento, el número de diferencias de AOD trazables supera con creces al número de diferencias no trazables. Hay que señalar, sin embargo, que en estos gráficos, el número de diferencias de AOD contenidas en cada intervalo FCS por debajo del $20 \%$ es mucho menor que el número de diferencias de AOD contenidas en cada intervalo FCS cuando este supera el $20 \%$. Este número es especialmente grande cuando se supera el $80 \%$ de cielo despejado (FCS $>80 \%$ ). Esto es debido a que, al nivel del Observatorio Atmosférico de Izaña existe una mayor frecuencia, durante todo el año, de cielos despejados o de días con poca presencia de nubes frente a días nubosos o cubiertos.

Como ya se ha explicado, todos estos porcentajes son estimativos y darían idea de la máxima influencia de la nubosidad en la no trazabilidad en los respectivos canales porque de ellos habría que extraer y descartar aquellos registros en los que el motivo de la no trazabilidad no haya sido la presencia de nubes sino alguna otra causa ajena al proceso de medida o de índole no meteorológica y que desconocemos.

De este estudio preliminar podemos concluir que, efectivamente, en los días con mayor nubosidad existe un mayor porcentaje de datos no trazables. Esto es probablemente debido a diferencias en los algoritmos de detección de nubes y al diferente campo de visión de los instrumentos GAW-PFR y AERONET-CIMEL. 


\subsection{Influencia de otros factores}

Como ejemplo de esto que acabamos de decir, en la Figura 9, se representan para el canal de 380nm, junto a las diferencias de AOD (en color magenta), aquellas no trazables tanto para días despejados (en azul, Figura 9 lzq.) como no despejados (en negro, Figura 9 Dcha.), y en ambos casos, con errores en la calibración inferiores al $1 \%$.
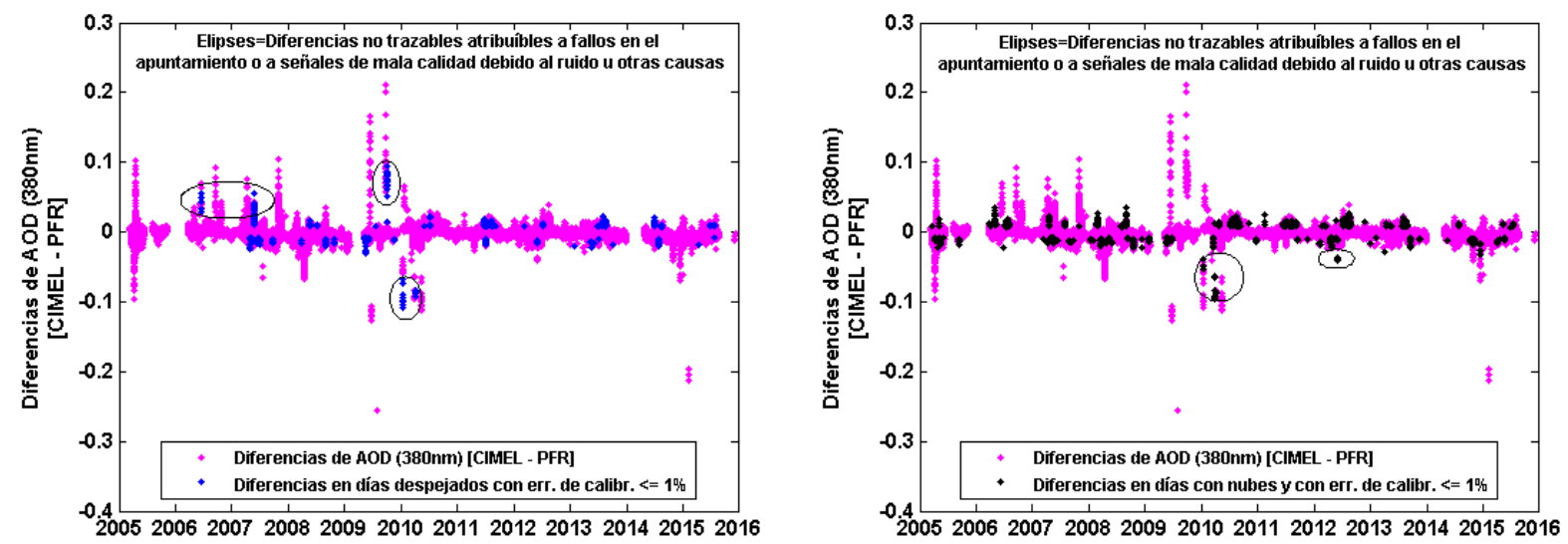

Figura 9: Izq.: En azul, diferencias no trazables de AOD en $380 \mathrm{~nm}$ para cielos despejados y con errores de calibración estimados menores del 1\%. Dcha.: En negro, diferencias no trazables de AOD en $380 \mathrm{~nm}$ para cielos con presencia de nubes y con errores de calibración estimados menores del I\%. Dentro de las elipses se encuentran aquellas diferencias que atribuimos a otras causas diferentes a las nubes (señales de baja calidad debido a ruido excesivo, obstáculos o fallos del sistema). Observatorio Atmosférico de Izaña, 2005 - 2015.

En la Figura 9 de la izquierda (cielos despejados), las causas de la no trazabilidad no pueden ser ni las nubes ni la calibración. Las diferencias de mayor magnitud (incluidas dentro de las elipses) son probablemente debidas a fallos en el apuntamiento al sol de los fotómetros. Las diferencias más pequeñas las atribuimos a errores debidos al cálculo del AOD. En la gráfica de la derecha, las diferencias no trazables corresponden a días con presencia de nubes y errores de calibración inferiores al I\% por lo que atribuimos, las de pequeña magnitud, a errores en el cálculo de AOD y las más grandes (englobadas en elipses) son probablemente debidas a una deficiente detección de nubes.

\section{Capítulo 3. Conclusiones y recomendaciones}

El análisis efectuado en este trabajo sobre la trazabilidad en el AOD evaluado hasta la fecha en el Observatorio Atmosférico de Izaña entre 2005 y 2015 por las redes GAW-PFR y AERONET-CIMEL en su versión 2 , nos permite llegar a las siguientes conclusiones:

I. El criterio para la trazabilidad del AOD al $95 \%$ definido por la OMM se cumple sobradamente para el canal de $870 \mathrm{~nm}$ con más del $97 \%$ de trazabilidad, y prácticamente se cumple para 440 y $500 \mathrm{~nm}$ con un $94.49 \%$ y un $94.26 \%$, respectivamente. La menor trazabilidad es la del canal de $380 \mathrm{~nm}$ que rebasa el umbral del $91 \%$.

2. La causa principal de la no trazabilidad en el canal de $870 \mathrm{~nm}$ está probablemente asociada a la calibración de alguno o de los dos equipos que se comparan. Esta causa explica el $37 \%$ de las diferencias no trazables analizadas en dicho canal (Figura 2). Para el resto de los canales de longitud de onda inferior, la causa principal de la no trazabilidad es el error relacionado con el cálculo del AOD y se sitúa entre el $36 \%$ y el $45 \%$ de las diferencias no trazables analizadas.

3. La absorción por $\mathrm{NO} 2$ en los canales por debajo de los $700 \mathrm{~nm}$, y en especial por debajo de $500 \mathrm{~nm}$, influye en la trazabilidad del $A O D$ en un orden de magnitud máximo de $10^{-3}$, que es el mismo orden de magnitud en el que se encuentran definidos los límites de trazabilidad. La consideración de la extinción debida al componente $\mathrm{NO} 2$ en tales canales podría ser significativa en zonas altamente contaminadas con elevado contenido de NO2 troposférico. En estos casos, sería recomendable que, en la evaluación del AOD para canales por debajo de $700 \mathrm{~nm}$, se tenga presente dicha absorción. 
4. La utilización de una misma fuente de datos de entrada para la presión, el ozono y el NO2 en los cálculos de AOD mejora su trazabilidad aproximadamente el $1 \%$ en el canal de $380 \mathrm{~nm}$ y menos del $0.5 \%$ en los otros dos canales por debajo de $870 \mathrm{~nm}$. Para este último, no se produce ninguna mejora.

5. La nubosidad tiene una influencia estimada en la no trazabilidad del AOD algo superior al $32 \%$ en todos los canales por debajo de $870 \mathrm{~nm}$. En este último, la influencia es mínima, reduciéndose a poco más del $2.5 \%$. Estas son estimaciones ya que no disponemos de herramientas adecuadas para discernir con precisión situaciones en las que únicamente haya polvo, o sólo nubes estratiformes estables y delgadas (como cirrostratos), o mezcla de nubes estratiformes y estables con polvo en cantidades variables.

En resumen, con este trabajo hemos puesto de manifiesto que la trazabilidad entre los AOD obtenidos en longitudes de ondas similares a partir de medidas extrapoladas y de medidas realizadas en canales originales de los fotómetros de dos redes completamente diferentes tanto por su diseño, óptica, y metodologías de calibración y procesamiento de datos de AOD, son excelentes.

\section{Agradecimientos}

Los autores agradecen a PMOD/WRC y AERONET los datos proporcionados y utilizados en este trabajo así como las calibraciones de los fotómetros Cimel por parte de AERONET-Europe TNA en el marco del proyecto europeo FP7 ACTRIS n²62254. Este trabajo forma parte de las actividades del "WMO-CIMO Testbed for Aerosols and Water Vapor Remote Sensing Instruments (Izana, Spain)”.

\section{Referencias}

Basart, S., Pérez, C., Cuevas, E., Baldasano, J. M., and Gobbi, G. P.: Mineral dust characterization for North of Africa, Northeastern Atlantic, Mediterranean Basin and Middle East from direct-sun AERONET observations, Atmos. Chem. Phys., 9, 82658282, doi:10.5194/acp-9-8265-2009, 2009.

Barreto, A., Cuevas, E., Granados-Muñoz, M. J., Alados-Arboledas, L., Romero, P. M., Gröbner, J., Kouremeti, N., Almansa, A. F., Stone, T., Sorokin, M., Holben, B., Canini, M., and Yela, M.: The new sun-sky-lunar Cimel CE3I8-T multiband photometer - a comprehensive performance evaluation , Atmos. Meas. Tech., 9, 631-654, 2016 doi: 10.5 I 94/amt-9-63|-2016.

Bodhaine, B. A., Wood, N. B., Dutton, E. G., and Slusser, J. R.: On Rayleigh Optical Depth Calculations, J. Atmos. and Ocean. Tech., 16, I854-186I, 1999.

Cachorro, V. E., Romero, P. M., Toledano C., Cuevas E., and De-Frutos A. M.: The fictitious diurnal cycle of aerosol optical depth: A new approach for "in situ" calibration and correction of AOD data series, Geophys. Res. Lett., 3I, LI2106, doi:10.1029/2004GL01965I, 2004.

Chubarova, N. Y., Poliukhov, A. A., and Gorlova, I. D.: Long-term variability of aerosol optical thickness in Eastern Europe over 200I-2014 according to the measurements at the Moscow MSU MO AERONET site with additional cloud and NO2 correction, Atmos. Meas. Tech., 9, 3।3-334, doi:10.5194/amt-9-3।3-2016, 2016.

Cuevas, E., González, Y., Rodríguez, S., Guerra, J. C., Gómez-Peláez, A. J., Alonso-Pérez, S., Bustos, J., and Milford, C.: Assessment of atmospheric processes driving ozone variations in the subtropical North Atlantic free troposphere, Atmos. Chem. Phys., 13, 1973-1998, doi:10.5194/acp-13-1973-2013, 2013.

Eck, T. F., Holben, B. N., Reid, J. S., Dubovik, O., Smirnov, A., O’Neill, N. T., Slutsker, I. and Kinne, S.: Wavelength dependence of the optical depth of biomass burning, urban, and desert dust aerosols, J. Geophys. Res. Atmospheres, 104(D24), 31333-31349, doi: 10.1029/1999JD900923, 1999. 
Fröhlich C. and Shaw G. E.: New determination of Rayleigh scattering in the terrestrial atmosphere, Applied Optics, 19, I773-1775, 1980.

García, R. D., Cuevas, E., García, O. E., Cachorro, V. E., Pallé, P., Bustos, J. J., Romero-Campos, P. M., and de Frutos, A. M.: Reconstruction of global solar radiation time series from 1933 to 2013 at the Izaña Atmospheric Observatory, Atmos. Meas. Tech., 7, 3I39-3I50, doi:I0.5I94/amt-7-3I39-20I4, 2014.

Gueymard, C. A.: SMARTS2, A Simple Model of the Atmospheric Radiative Transfer of Sunshine: Algorithms and performance assessment, Florida Solar Energy Center Cocoa FL / University of Central Florida, FSEC-PF-270-95, December 1995.

Holben, B. N., Eck, T. F., Slutsker, I., Tanré, D., Buis, J. P., Setzer, A., Vermote, E., Reagan, J. A., Kaufman, Y. J., Nakajima, T., Lavenu, F., Jankowiak, I. and Smirnov, A.: AERONET-A Federated Instrument Network and Data Archive for Aerosol Characterization, Remote Sens. Environ., 66(I), I - 16, doi:http://dx.doi.org/10.1016/S00344257(98)0003 I-5, 1998.

Kasten, F., Arch.Meteo.Geophys.Bioklim Ser.B, Bd.I4, 206-223, 1966.

Kasten, F. and Young, A. T.: Revised optical air mass tables and approximation formula, Appl. Opt., 28, 4735-4738, 1989.

Komhyr, W. D.: Dobson operations handbook, NOAA/CMDL, 1980.

Komhyr, II'. D., Grass, K. D., and Leonard, R. K.: Dobson Spectrophotometer 83: a standard for total ozone measurements, 1962-1987. J. Geophys. Res. 94:9847-986I, 1989.

Rodríguez, S., Alastuey, A., Alonso-Pérez, S., Querol, X., Cuevas, E., Abreu-Afonso, J., Viana, M., Pérez, N., Pandolfi, M., and de la Rosa, J.: Transport of desert dust mixed with North African industrial pollutants in the subtropical Saharan Air Layer, Atmos. Chem. Phys., I I, 6663-6685, doi:I0.5I94/acp-I I-6663-20 I I, 20 II.

Romero, P. M., y E. Cuevas, Variación diurna del espesor óptico de aerosoles: ¿"ficción" o realidad ?, $3^{\mathrm{a}}$ Asamblea Hispano-Portuguesa de Geodesia y Geofísica, Valencia, 4-8 febrero, 2002.

Smirnov, A. et al.: Cloud screening and quality control algorithms for Aeronet database. Rem. Sensing Environment, 73, 337-349, 2000.

Wehrli, C.: Calibrations of filter radiometers for determination of atmospheric optical depth, Metrologia, 37(5), 4I9-422, doi:10.1088/0026-1394/37/5/16, 2000.

Wehrli, C.: GAW-PFR: A Network of Aerosol Optical Depth Observations with Precision Filter Radiometers, in: WMO/GAW Experts Workshop on a Global Surface-Based Network for Long Term Observations of Column Aerosol Optical Properties, GAW Report No. 162, WMO TD No. 1287, [online] Available from: http://library.wmo.int/pmb_ged/wmo-td_1287.pdf, 2005.

Wehrli, C.: Precision Filter Radiometer. Documentation, (ftp://ftp.pmodwrc.ch/pub/worcc/PFRManual.pdf), 2008.

WMO: WMO/GAW Experts Workshop on a Global Surface-Based Network for Long Term Observations of Column Aerosol Optical Properties, edited by: Baltensperger, U., Barrie, L., and Wehrli, C., GAW No. I62, WMO/TD-No. I287. Davos (Switzerland), 2005.

WMO: Guide to Meteorological Instruments and Methods of Observation, WMO No 8, 2014 edition, ISBN 978-9263-10008-5, 2014.

Young, A. T.: Revised depolarization correction for atmospheric extinction, Applied Optics, 19, 3427-3428, 1980. 